\title{
Radiative neutrino mass and Majorana dark matter within an inert Higgs doublet model
}

\author{
Amine Ahriche, ${ }^{1,2, *}$ Adil Jueid, ${ }^{3,4, \dagger}$ and Salah Nasri ${ }^{5,2, \$}$ \\ ${ }^{1}$ Department of Physics, University of Jijel, PB 98 Ouled Aissa, Jijel DZ-18000, Algeria \\ ${ }^{2}$ The Abdus Salam International Centre for Theoretical Physics, Strada Costiera 11, Trieste I-34014, Italy \\ ${ }^{3}$ INPAC, Shanghai Key Laboratory for Particle Physics and Cosmology, \\ Department of Physics and Astronomy, Shanghai Jiao Tong University, Shanghai 200240, China \\ ${ }^{4}$ Département des Mathématiques, Faculté des Sciences et Techniques, \\ Université Abdelmalek Essaadi, Tanger, Morocco \\ ${ }^{5}$ Department of physics, United Arab Emirates University, Al-Ain, UAE
}

(Received 15 October 2017; revised manuscript received 14 March 2018; published 14 May 2018)

\begin{abstract}
We consider an extension of the standard model (SM) with an inert Higgs doublet and three Majorana singlet fermions to address both origin and the smallness of neutrino masses and dark matter (DM) problems. In this setup, the lightest Majorana singlet fermion plays the role of DM candidate and the model parameter space can be accommodated to avoid different experimental constraints such as lepton flavor violating processes and electroweak precision tests. The neutrino mass is generated at one-loop level a la Scotogenic model and its smallness is ensured by the degeneracy between the $C P$-odd and $C P$-even scalar members of the inert doublet. Interesting signatures at both leptonic and hadronic colliders are discussed.
\end{abstract}

DOI: 10.1103/PhysRevD.97.095012

\section{INTRODUCTION}

The discovery of the Higgs particle at the LHC in 2012 [1], validated the standard model (SM) of particle physics. Although the SM has been very successful in describing and explaining the nongravitational fundamental interactions between elementary particles, there are still open questions that it does not answer. For instance, the observation of neutrino oscillations in solar, atmospheric, reactor and accelerator experiments confirmed that neutrino have tiny non zero mass, unlike in the SM where they are strictly massless. Another issue that the SM does not account for is the existence of dark matter (DM) inferred from different astronomical and cosmological observations. Thus, going beyond the SM seems to be necessary in order to address these problems.

The most popular mechanism for explaining the smallness of neutrino mass is the seesaw mechanism, where a massive right handed neutrino (RHN) couples to the lepton and the Higgs doublets fields, and which at low energies induces the effective dimension five operator

\footnotetext{
*aahriche@daad-alumni.de †adil.jueid@sjtu.edu.cn

snasri@uaeu.ac.ae
}

Published by the American Physical Society under the terms of the Creative Commons Attribution 4.0 International license. Further distribution of this work must maintain attribution to the author(s) and the published article's title, journal citation, and DOI. Funded by SCOAP ${ }^{3}$.

$$
\mathcal{L}_{\text {eff }}^{(5)}=\frac{\kappa_{i j}}{\Lambda}\left(\overline{L_{i}^{c} i \sigma_{2} H}\right)\left(L_{j}^{T} i \sigma_{2} H\right)+\text { H.c. },
$$

where $\Lambda$ is the scale of new physics which is of order the RH neutrino mass, $\kappa_{i j}$ is a product of Yukawa couplings, $L$ and $L^{c}$ are the Lepton $S U(2)_{L}$ doublet and its charge conjugate, respectively, and $H$ is the Higgs doublet. However, on the basis of naturalness, the scale $\Lambda$ needs to be of order the grand unified theory (GUT) scale, making it impossible to probe it in high energy laboratory experiments. One way to lower the scale of the new physics is by generating neutrino masses radiatively where their smallness can be naturally explained by the loop suppression factor(s) and the Yukawa couplings. This can be realized at one loop [2], two loops [3], three loops $[4,5]$, or four loops [6]. In [7] it has been shown that there is a class of three loop neutrino mass generation models, where the new particles that enter in the neutrino loop can be promoted to triplets [7], quintuplet [8], septuplets [9], and within a scale-invariant framework [10]. An interesting features of these class of models is that the scale of new physics can be of order $\mathrm{TeV}$, making them testable at high energy colliders [11,12] (For a review, see Ref. [13]). Moreover, the electroweak phase transition can be strongly first order, an essential ingredient for successful electroweak baryogenesis [14].

One of the simplest extensions of the scalar sector of the SM is the inert Higgs doublet model (IHDM) where one add an extra scalar doublet, $\Phi$, and assumes that there is a discrete $Z_{2}$ symmetry under which $\Phi$ is odd whereas the SM fields are even [15]. The phenomenology of the 
(IHDM) has been studied extensively in the literature [16]. The discrete symmetry $Z_{2}$ has the following features: (i) absence of the flavor changing neutral currents at tree level [17], (ii) the scalar of the inert doublet does not have interactions with active fermions and therefore its neutral component, denoted by $H^{0}$, can be a good candidate for DM. It has been shown that if $H^{0}$ is lighter than about $\leq 50 \mathrm{GeV}$, the null results from DM direct detection experiment implies that annihilation cross section of the neutral inert scalar into SM light fermions is highly suppressed, giving too large relic density to what is observed by Planck experiment. ${ }^{1}$ In the mass range $140 \mathrm{GeV}<m_{H^{0}} \leq 550 \mathrm{GeV}$, the annihilation into $W^{+} W^{-}$is too large, rendering the relic density too small to be compatible with the astrophysical observations. There are three mass regions where the inert scalar can be a viable DM candidate: (i) $m_{H^{0}} \simeq m_{h} / 2$, with $m_{h}$ is the SM Higgs mass, corresponding to the annihilation via the s-channel resonance due to Higgs exchange [19], (ii) $m_{H^{0}}$ around the mass of the $W$ gauge boson, where the annihilation is into the three-body final state $W W^{*} \rightarrow W f f^{\prime}$ [20], and (iii) $m_{H^{0}} \sim \mathrm{TeV}$ with scalar couplings of order unity [21]. One possible way to produce the correct relic density and not being in conflict with DM direct detection experiment is by extending this model with heavy $S U(2)_{L}$ singlet vectorlike charged leptons [19].

In the present work, we consider the SM extended by an inert doublet and three RHNs [22] in order to address both neutrino mass and DM problem. We will investigate the case where the lightest Majorana singlet fermion plays the role of DM candidate instead of the neutral $C P$-odd or $C P$ even scalars ${ }^{2}$ We will show that the model parameter space can be accommodated to avoid different experimental constraints such as lepton flavor violating processes and electroweak precision tests. In this setup, the neutrino mass smallness is ensured by making the splitting between the $C P$-even and $C P$-odd scalars very tiny. We will study different phenomenological aspects of the model and investigate the different regions of the parameter space that fulfill various theoretical and experimental constraints. In addition, we discuss the signatures for probing the model at both leptonic and hadronic colliders are discussed.

The paper is organized as follows: in Sec. II, we highlight the model, its parameters and the different constraints under which the model is subject to. In Sec. III, we present some selected results. In Sec. IV, we discuss briefly the possible signatures of this model at hadronic and leptonic collider machines. In Sec. V, we give our conclusion.

\footnotetext{
${ }^{1}$ This can be avoided if there are other annihilation channels as in [18].

${ }^{2}$ In this radiative neutrino mass model, the allowed mass range for inert scalar dark matter remains very similar to the case of pure IHDM [19].
}

\section{THE MODEL: PARAMETERS AND CONSTRAINTS}

\section{A. The model and mass spectrum}

Here we consider the extension of the SM with an inert doublet $\Phi$ and three singlet Majorana fermions $N_{i} \sim(1,1,0), i=1,2,3$, both odd under a discrete $Z_{2}$ symmetry. The relevant terms for generating neutrino mass at one loop are

$$
\mathcal{L} \supset h_{i j} \bar{L}_{i} \epsilon \Phi N_{j}+\frac{1}{2} M_{i} \bar{N}_{i}^{C} N_{i}+\text { H.c. }
$$

where $\bar{L}_{i}$ is the left-handed lepton doublet and $\epsilon=i \sigma_{2}$ is an antisymmetric tensor. The scalar potential can be written as

$$
\begin{aligned}
V= & -\mu_{1}^{2}|H|^{2}+\mu_{2}^{2}|\Phi|^{2}+\frac{\lambda_{1}}{6}|H|^{4}+\frac{\lambda_{2}}{6}|\Phi|^{4}+\lambda_{3}|H|^{2}|\Phi|^{2} \\
& +\frac{\lambda_{4}}{2}\left|H^{\dagger} \Phi\right|^{2}+\frac{\lambda_{5}}{4}\left[\left(H^{\dagger} \Phi\right)^{2}+\text { H.c. }\right],
\end{aligned}
$$

and the SM Higgs and the inert doublets can be represented as

$$
H=\left(\begin{array}{c}
G^{+} \\
\frac{1}{\sqrt{2}}\left(v+h+i G^{0}\right)
\end{array}\right), \quad \Phi=\left(\begin{array}{c}
H^{+} \\
\frac{1}{\sqrt{2}}\left(H^{0}+i A^{0}\right)
\end{array}\right) .
$$

Keeping in mind that all field dependent masses are written in the from $m_{i}^{2}(h)=\mu_{i}^{2}+\frac{1}{2} \alpha_{i} h^{2}$, the parameters $\lambda_{1}$ and $\mu_{1}^{2}$ in (3) can be eliminated in favor of the SM Higgs mass and its vacuum expectation value $(v=246 \mathrm{GeV})$, which is considered at one-loop level as

$$
\begin{aligned}
& \lambda_{1}=\frac{3 m_{h}^{2}}{v^{2}}-\frac{3}{32 \pi^{2}} \sum_{i=\text { all }} n_{i} \alpha_{i}^{2} \log \frac{m_{i}^{2}}{m_{h}^{2}}, \\
& \mu_{1}^{2}=\frac{1}{6} \lambda_{1} v^{2}+\frac{1}{32 \pi^{2}} \sum_{i=\text { all }} n_{i} \alpha_{i} m_{i}^{2}\left(\log \frac{m_{i}^{2}}{m_{h}^{2}}-1\right) .
\end{aligned}
$$

Here, the one-loop contribution to the effective potential is defined a la $\overline{D R}$ scheme [23], where the renormalization scale is taken to be the Higgs mass ${ }^{3}$ After the spontaneous symmetry breaking, we are left with two $C P$-even scalars $\left(h, H^{0}\right)$, one $C P$-odd scalar $A^{0}$ and a pair of charged scalars $H^{ \pm}$. Their tree-level masses are given by:

$m_{H^{ \pm}}^{2}=\mu_{2}^{2}+\frac{1}{2} \lambda_{3} v^{2}, \quad m_{H^{0}, A^{0}}^{2}=m_{H^{ \pm}}^{2}+\frac{1}{4}\left(\lambda_{4} \pm \lambda_{5}\right) v^{2}$.

Note that the $Z_{2}$ symmetry forbids the term $Y_{i j} \bar{L}_{i} H N_{j}$ in the Lagrangian, and hence rendering neutrinos massless at the tree level. However they get mass at the one loop level (see Fig. 1), given ${ }^{4}$

\footnotetext{
${ }^{3}$ The one-loop corrections in (5) could be important if the coupling combinations $\alpha_{i}=\lambda_{3}, \lambda_{3}+\lambda_{4} / 2 \pm \lambda_{5} / 2$ are large, and/ or the inert scalar doublet scalars are heavy.

${ }^{4}$ Note that we use the corrected overall factor $1 / 32$ [24] instead of the $1 / 16$ given in [22].
} 


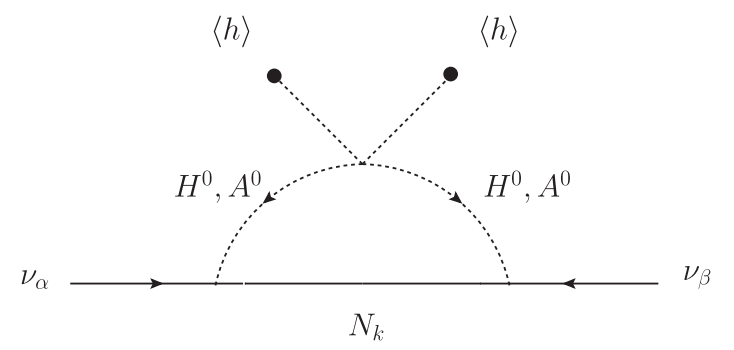

FIG. 1. Feynman diagram responsible for the neutrino mass.

$m_{\alpha \beta}^{(\nu)}=\sum_{k} \frac{h_{\alpha k} h_{\beta k} M_{k}}{32 \pi^{2}}\left[\frac{m_{H^{0}}^{2}}{m_{H^{0}}^{2}-M_{k}^{2}} \ln \frac{m_{H^{0}}^{2}}{M_{k}^{2}}-\frac{m_{A^{0}}^{2}}{m_{A^{0}}^{2}-M_{k}^{2}} \ln \frac{m_{A^{0}}^{2}}{M_{k}^{2}}\right]$.

If the mass splitting $m_{H^{0}}^{2}-m_{A^{0}}^{2}=\frac{1}{2}\left|\lambda_{5}\right| v^{2}$ is small with respect to the average $\bar{m}^{2}=\left(m_{H^{0}}^{2}+m_{A^{0}}^{2}\right) / 2$, which is favored by the electroweak precision measurements, then the expression of the mass matrix elements is simplified to

$$
m_{\alpha \beta}^{(\nu)} \simeq \frac{\left|\lambda_{5}\right| v^{2}}{16 \pi^{2}} \sum_{k} \frac{h_{\alpha k} h_{\beta k} M_{k}}{\bar{m}^{2}-M_{k}^{2}}\left[1-\frac{M_{k}^{2}}{\bar{m}^{2}-M_{k}^{2}} \ln \frac{\bar{m}^{2}}{M_{k}^{2}}\right] .
$$

The neutrino mass matrix elements in (8) can be related to the elements of the Pontecorvo-Maki-Nakawaga-Sakata (PMNS) mixing matrix [25] elements. We parametrize the latter as

$$
U_{\nu}=\left(\begin{array}{ccc}
c_{12} c_{13} & c_{13} s_{12} & s_{13} e^{-i \delta_{d}} \\
-c_{23} s_{12}-c_{12} s_{13} s_{23} e^{i \delta_{d}} & c_{12} c_{23}-s_{12} s_{13} s_{23} e^{i \delta_{d}} & c_{13} s_{23} \\
s_{12} s_{23}-c_{12} c_{23} s_{13} e^{i \delta_{d}} & -c_{12} s_{23}-c_{23} s_{12} s_{13} e^{i \delta_{d}} & c_{13} c_{23}
\end{array}\right) \times U_{m}
$$

with $\delta_{d}$ the Dirac phase and $U_{m}=\operatorname{diag}\left(1, e^{i \theta_{\alpha} / 2}, e^{i \theta_{\beta} / 2}\right)$ encoding the Majorana phase dependence. The shorthand $s_{i j} \equiv \sin \theta_{i j}$ and $c_{i j} \equiv \cos \theta_{i j}$ refers to the mixing angles. For our numerical scans (discussed below) we fit to the best-fit experimental values for the mixing angles and mass-squared differences: $\quad s_{12}^{2}=0.320_{-0.017}^{+0.016}, \quad s_{23}^{2}=0.43_{-0.03}^{+0.03}, \quad s_{13}^{2}=$ $0.025_{-0.003}^{+0.003},\left|\Delta m_{13}^{2}\right|=2.55_{-0.09}^{+0.06} \times 10^{-3} \mathrm{eV}^{2}$ and $\Delta m_{21}^{2}=$ $7.62_{-0.19}^{+0.19} \times 10^{-5} \mathrm{eV}^{2}$ [26]. Furthermore, we require that the contribution to neutrinoless double beta decay in this model satisfies the current bound. Within these ranges, one determines the parameter space where viable neutrino masses and mixing occur in the model.

\section{B. Theoretical and experimental constraints}

Here, we discuss different theoretical and experimental constraints on the model parameters.

Theoretical Constraints: The parameters of the scalar potential have to satisfy these theoretical constraints:

(i) Perturbativity: all the quartic couplings of the physical fields should be less than $4 \pi$, i.e.,

$$
\lambda_{1}, \lambda_{2},\left|\lambda_{3}+\frac{1}{2} \lambda_{4}\right|,\left|\lambda_{3}+\frac{1}{2}\left(\lambda_{4} \mp \lambda_{5}\right)\right| \leq 4 \pi
$$

(ii) Vacuum stability: the scalar potential is required to be bounded from below in all the directions of the field space. In both field planes $h-H^{0}$ and $G^{0}-A^{0}$ the following the condition must be satisfied [27]

$$
\lambda_{1}, \lambda_{2}, \lambda_{3}+\frac{1}{2}\left(\lambda_{4}+\lambda_{5}\right)+\sqrt{\lambda_{1} \lambda_{2}}>0,
$$

whereas in the plane $H^{ \pm}-G^{\mp}$, we find

$$
\lambda_{3}+\frac{1}{2} \lambda_{4}+\frac{2}{3} \sqrt{\lambda_{1} \lambda_{2}}>0
$$

In addition, we should consider the condition

$$
\frac{\mu_{1}^{2}}{\sqrt{\lambda_{1}}} \geq-\frac{\mu_{2}^{2}}{\sqrt{\lambda_{2}}},
$$

which is required to guarantee that the inert vacuum is the global minimum [28].

(iii) Perturbative unitarity: We demand that the perturbative unitarity is preserved in variety of processes involving scalars or gauge bosons at high energy. At high energies, the equivalence theorem replaces the $W$ and $Z$ bosons by the Goldstone bosons. Computing the decay amplitudes for these processes, one finds a set of 4 matrices with quartic couplings as their entries [29]. The diagonalization of the scattering matrix gives the following eigenvalues

$$
\begin{aligned}
e_{1,2} & =\lambda_{3} \pm \frac{\lambda_{4}}{2}, \quad e_{3,4}=\lambda_{3} \pm \frac{\lambda_{5}}{2}, \\
e_{5,6} & =\lambda_{3}+\lambda_{4} \pm \frac{3}{2} \lambda_{5}, \\
e_{7,8} & =-\frac{\lambda_{1}+\lambda_{2}}{6} \pm \sqrt{\left(\frac{\lambda_{1}+\lambda_{2}}{6}\right)^{2}+\frac{\lambda_{4}^{2}}{4}} \\
e_{9,10} & =-\frac{\lambda_{1}+\lambda_{2}}{2} \pm \sqrt{9\left(\frac{\lambda_{1}-\lambda_{2}}{6}\right)^{2}+\left(2 \lambda_{3}+\frac{\lambda_{4}}{2}\right)^{2}}, \\
e_{11,12} & =-\frac{\lambda_{1}+\lambda_{2}}{6} \pm \sqrt{\left(\frac{\lambda_{1}-\lambda_{2}}{6}\right)^{2}+\frac{\lambda_{5}^{2}}{4}} .
\end{aligned}
$$


We require that the largest eigenvalue of these matrices to be smaller than $4 \pi$.

Experimental constraints

(i) Gauge bosons decay widths: in order to keep the $W$ and $Z$ gauge bosons decay modes unmodified, one needs to impose the following conditions:

$$
\begin{aligned}
m_{H^{0}}+m_{A^{0}}>M_{Z}, & m_{H^{ \pm}}+m_{A^{0}}>M_{W}, \\
2 m_{H^{ \pm}}>M_{Z}, & m_{H^{ \pm}}+m_{H^{0}}>M_{W} .
\end{aligned}
$$

(ii) Lepton flavor violation (LFV) processes: in this model, LFV decay processes arise at one-loop level with the exchange of $H^{ \pm}$and $N_{k}$ particles. The branching ratio of the decay $\ell_{\alpha} \rightarrow \ell_{\beta}+\gamma$ due to the contribution of the interactions (2) is [30].

$$
\begin{aligned}
& \operatorname{Br}\left(\ell_{\alpha} \rightarrow \ell_{\beta}+\gamma\right) \\
& \quad=\frac{3 \alpha v^{4}}{32 \pi m_{H^{ \pm}}^{4}}\left|\sum_{i=1}^{3} h_{\beta i}^{*} h_{\alpha i} F\left(M_{i}^{2} / m_{H^{ \pm}}^{2}\right)\right|^{2},
\end{aligned}
$$

where $\alpha=e^{2} / 4 \pi$ is the electromagnetic fine structure constant and $F(x)=\left(1-6 x+3 x^{2}+2 x^{3}-\right.$ $\left.6 x^{2} \log x\right) / 6(1-x)^{4}$. We will consider also the LFV decays $\ell_{\alpha} \rightarrow \ell_{\beta} \ell_{\beta} \ell_{\beta}$, where their branching ratio formulas are given in [30]. In our numerical scan, we will impose all the experimental limits on both $\operatorname{Br}\left(\ell_{\alpha} \rightarrow \ell_{\beta}+\gamma\right)$ and $\operatorname{Br}\left(\ell_{\alpha} \rightarrow \ell_{\beta} \ell_{\beta} \ell_{\beta}\right)$ [31].

(iii) The electroweak precision tests: while taking $\Delta U=$ 0 in our analysis, the oblique parameters can be written in our model as [32]

$$
\begin{aligned}
& \Delta T=\frac{1}{16 \pi s_{\mathrm{w}}^{2} M_{W}^{2}}\left\{F\left(m_{H^{0}}^{2}, m_{H^{ \pm}}^{2}\right)+F\left(m_{A^{0}}^{2}, m_{H^{ \pm}}^{2}\right)-F\left(m_{H^{0}}^{2}, m_{A^{0}}^{2}\right)\right\}, \\
& \Delta S=\frac{1}{24 \pi}\left\{\left(2 s_{\mathrm{w}}^{2}-1\right)^{2} G\left(m_{H^{ \pm}}^{2}, m_{H^{ \pm}}^{2}, M_{Z}^{2}\right)+G\left(m_{H^{0}}^{2}, m_{A^{0}}^{2}, M_{Z}^{2}\right)+\ln \left(\frac{m_{H^{0}}^{2} m_{A^{0}}^{2}}{m_{H^{ \pm}}^{4}}\right)\right\},
\end{aligned}
$$

where $s_{\mathrm{W}} \equiv \sin \theta_{W}$, with $\theta_{W}$ is the Weinberg mixing angle, and the functions $F$ and $G$ are loop integrals that are given in the literature [32].

(iv) The ratio $R_{\gamma \gamma}$ : The existence of the charged scalar $H^{ \pm}$modifies the value of the branching ratio $\mathcal{B}(h \rightarrow \gamma \gamma)$, which both ATLAS and CMS collaborations have reported their combined results on the ratio $R_{\gamma \gamma}:=\mathcal{B}(h \rightarrow \gamma \gamma) /$ $\mathcal{B}(h \rightarrow \gamma \gamma)^{\mathrm{SM}}=1.09 \pm 0.12$ [33]. In the model we are considering, $R_{\gamma \gamma}$ reads

$$
R_{\gamma \gamma}=\left|1+\frac{\lambda_{3} v^{2}}{2 m_{H^{ \pm}}^{2}} \frac{A_{0}^{\gamma \gamma}\left(\frac{m_{h}^{2}}{4 m_{H^{ \pm}}^{2}}\right)}{A_{1}^{\gamma \gamma}\left(\frac{m_{h}^{2}}{4 M_{W}^{2}}\right)+N_{c} Q_{t}^{2} A_{1 / 2}^{\gamma \gamma}\left(\frac{m_{h}^{2}}{4 m_{t}^{2}}\right)}\right|^{2},
$$

where the functions $A_{i}^{\gamma \gamma}$ are given in [34]. Another Higgs decay branching ratio that gets modified is $h \rightarrow \gamma Z$, with the signal strength is given by

$$
R_{\gamma Z}=\left|1-\frac{1-2 s_{\mathrm{w}}^{2}}{c_{\mathrm{w}}} \frac{\lambda_{3} v^{2}}{2 m_{H^{ \pm}}^{2}} \frac{A_{0}^{\gamma Z}\left(\frac{m_{h}^{2}}{4 m_{H^{ \pm}}^{2}}, \frac{M_{Z}^{2}}{4 m_{H^{ \pm}}^{2}}\right)}{A_{1}^{\gamma Z}\left(\frac{m_{h}^{2}}{4 M_{W}^{2}}, \frac{M_{Z}^{2}}{4 M_{W}^{2}}\right)+\frac{6-16 s_{\mathrm{w}}^{2}}{3 c_{\mathrm{w}}} A_{1 / 2}^{\gamma Z}\left(\frac{m_{h}^{2}}{4 m_{t}^{2}}, \frac{M_{Z}^{2}}{4 m_{t}^{2}}\right)}\right|^{2},
$$

where $c_{\mathrm{w}}^{2}=1-s_{\mathrm{w}}^{2}$ and the functions $A_{i}^{\gamma Z}$ are given in [34]. The branching ratio $h \rightarrow \gamma Z$ is not measured yet, but when it will be measured with a good precision, it can give a hint about the extra charged scalar whether it is a singlet or it belongs to a higher order multiplet.

(v) LEP direct searches of charginos and neutralinos: The search for the inert particles have never been done at colliders. However, their signature is very similar to those of neutralinos and charginos in supersymmetric models [35]. We take a conservative approach and impose the following lower bounds

$m_{H^{ \pm}}>113.5 \mathrm{GeV}, \quad \max \left\{m_{H^{0}}, m_{A^{0}}\right\}>100 \mathrm{GeV}$, where the last bound comes from a re-interpretation of neutralino searches at LEP [36] in the context of the IHDM.

(vi) Dark matter relic density: In this model, we are considering the lightest right handed neutrino to be the DM candidate. Its annihilation occurs onto SM neutrinos and charged leptons via t-channel diagrams mediated by the members of the Inert doublet. After computing the thermally averaged cross section [37], $\left\langle\sigma_{N_{1} N_{1}} v_{r}\right\rangle=\left\langle\sigma\left(N_{1} N_{1} \rightarrow \ell^{+} \ell^{-}, \nu \bar{\nu}\right) v_{r}\right\rangle$, the DM relic abundance can be expressed as [38]

$$
\Omega_{N_{1}} h^{2} \simeq \frac{3 \times 10^{-26} \mathrm{~cm}^{3} \mathrm{~s}^{-1}}{\left\langle\sigma_{N_{1} N_{1}} v_{r}\right\rangle}
$$




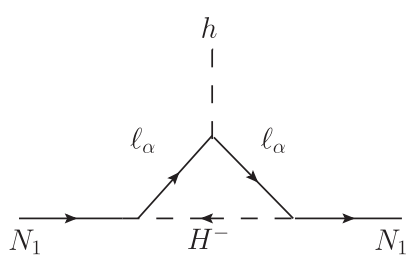

(a)

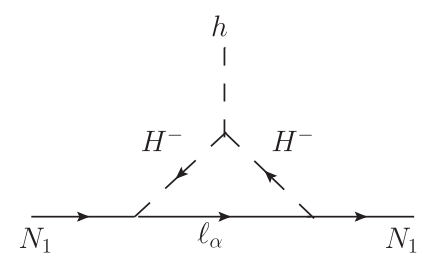

(b)

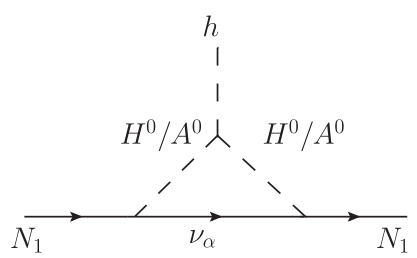

(c)

FIG. 2. Feynman diagrams that are responsible to the effective coupling $\tilde{y}_{h N_{1} \bar{N}_{1}}$.

which we require to be in agreement with the measured values by WMAP [39] and Planck [40] collaborations. The expression of the relic density in (21) is estimated without taking into account the coannihilation effect. Such effect can be important when $N_{2}$ and/or $N_{3}$ have masses very close to that of $N_{1}$, i.e., $\Delta_{i}=\left(M_{i}-M_{1}\right) / M_{1} \ll 1$, and hence one should not neglect it when computing the relic density. However, coannihilation with the inert members, such as the process $N_{1} H^{ \pm} \rightarrow \ell_{\alpha} \gamma$, is less important due to the smallness the electromagnetic coupling as compared to the Yukawa couplings $h_{i k}$.

In order to account for such effect, one substitute $\left\langle\sigma_{N_{1} N_{1}} v_{r}\right\rangle$ in (21) by the thermal average of the effective cross section [41]

$$
\begin{aligned}
\sigma_{\mathrm{eff}}\left(x_{f}\right)= & \sum_{i, k}^{3} \frac{2^{2}}{g_{\mathrm{eff}}^{2}}\left(1+\Delta_{i}\right)^{3 / 2}\left(1+\Delta_{k}\right)^{3 / 2} \\
& \times e^{-x_{f}\left(\Delta_{i}+\Delta_{k}\right)}\left\langle\sigma_{i k} v_{r}\right\rangle
\end{aligned}
$$

with

$$
g_{\mathrm{eff}}\left(x_{f}\right)=\sum_{i}^{3} 2\left(1+\Delta_{i}\right)^{3 / 2} e^{-x_{f} \Delta_{i}}
$$

Here, $x_{f}=M_{1} / T_{f}$ is the freeze-out parameter, $g_{\text {eff }}\left(x_{f}\right)$ accounts for the effective multiplicity at the freeze-out, and $\left\langle\sigma_{i k} v_{r}\right\rangle$ is the thermally averaged cross section of $N_{i} N_{k} \rightarrow \ell_{\alpha}^{-} \ell_{\beta}^{+}, \nu_{\alpha} \bar{\nu}_{\beta}$ at the freezeout. Here, the ratio

$$
\Delta_{i}=\frac{M_{i}-M_{1}}{M_{1}}
$$

is the relative mass difference. The cross section formulas for the processes $N_{i} N_{k} \rightarrow \ell_{\alpha}^{-} \ell_{\beta}^{+}, \nu_{\alpha} \bar{\nu}_{\beta}$ are given in Eq. (A2) in the Appendix.

(vii) Dark matter direct detection: Although $N_{1}$ does not couple directly to the Higgs boson or $Z$ gauge boson, it acquire an effective vertex $h N_{1} \bar{N}_{1}$ at one loop. In this case, the spin independent (SI) scattering cross section of $N_{1}$ off a nucleon $\mathcal{N}$ reads

$$
\begin{aligned}
& \sigma_{\mathrm{det}}\left(N_{1}+\mathcal{N} \rightarrow N_{1}+\mathcal{N}\right) \\
& =\frac{\tilde{y}_{h N_{1} \bar{N}_{1}}^{2}\left(m_{\mathcal{N}}-\frac{7}{9} m_{\mathcal{B}}\right)^{2} m_{\mathcal{N}}^{2} M_{1}^{2}}{4 \pi v^{2} m_{h}^{4}\left(m_{\mathcal{N}}+M_{1}\right)^{2}},
\end{aligned}
$$

where $m_{\mathcal{N}}$ and $m_{\mathcal{B}}$ are the nucleon and baryon masses in the chiral limit [42], and $\tilde{y}_{h N_{1} \bar{N}_{1}}$ is the induced one loop effective dark matter coupling to the Higgs boson. There are three generic contributions to the effective $\tilde{y}_{h N_{1} \bar{N}_{1}}$ coupling that lead to the SI interaction as are shown in Fig. 2.

In nonrelativistic limit and $m_{\ell_{\alpha}} \ll M_{1}$, the effective coupling can be approximated by $[43]^{5}$

$$
\begin{aligned}
\tilde{y}_{h N_{1} N_{1}}= & -\frac{\lambda_{3} v \sum_{\alpha}\left|h_{\alpha 1}\right|^{2}}{16 \pi^{2} M_{1}^{3}} \\
& \times\left(M_{1}^{2}+\left(m_{H^{ \pm}}^{2}-M_{1}^{2}\right) \ln \left(\frac{m_{H^{ \pm}}^{2}-M_{1}^{2}}{m_{H^{ \pm}}^{2}}\right)\right) \\
& -\frac{\left(\lambda_{3}+\lambda_{4} / 2\right) v \sum_{\alpha}\left|h_{\alpha 1}\right|^{2}}{16 \pi^{2} M_{1}^{3}} \\
& \times\left(M_{1}^{2}+\left(\bar{m}^{2}-M_{1}^{2}\right) \ln \left(\frac{\bar{m}^{2}-M_{1}^{2}}{\bar{m}^{2}}\right)\right),
\end{aligned}
$$

where $\bar{m}^{2}=\left(m_{H^{0}}^{2}+m_{A^{0}}^{2}\right) / 2$. In the above expression, the first [second] line corresponds to diagram (b), and the second line [(c)], whereas diagram (a) contribution is negligible since it is $m_{\ell_{\alpha}}^{2} / m_{H^{ \pm}}^{2}$ proportional. Thus, in our scan of the parameter space we will impose the recent bounds on the dark matternucleon scattering cross section from the LUX [44] and XENON1T [45] direct detection experiments.

\section{NUMERICAL ANALYSIS AND DISCUSSION}

The model contains 26 free parameters: 4 quartic couplings in the scalar potential $\lambda_{i}, i=2 \ldots 5,18$ (9 complex) Yukawa couplings $h_{i j}, i, j=1 \ldots 3,3$ RHNs masses $M_{k}, k=$ 1 ...3 and the squared-mass parameter $\mu_{2}^{2}$ of the inert Higgs. In our numerical analysis, we perform a scan of those parameters over the parameters ranges

$$
\begin{aligned}
0 & <\lambda_{2},\left|\lambda_{3,4}\right|<2, \\
1 \mathrm{GeV} & <M_{1}<M_{2}<M_{3}<2 \mathrm{TeV}, \\
-v^{2} & <\mu_{2}^{2}<7 v^{2}, \quad 10^{-5}<\left|h_{i j}\right|<1 .
\end{aligned}
$$

Taking into account all theoretical and experimental constraints mentioned in the previous section, we scan over the parameters range (27). Within this parameters range, one can estimate the effect of the one-loop corrections in (5)

\footnotetext{
${ }^{5}$ The exact formula is derived in Appendix B.
} 

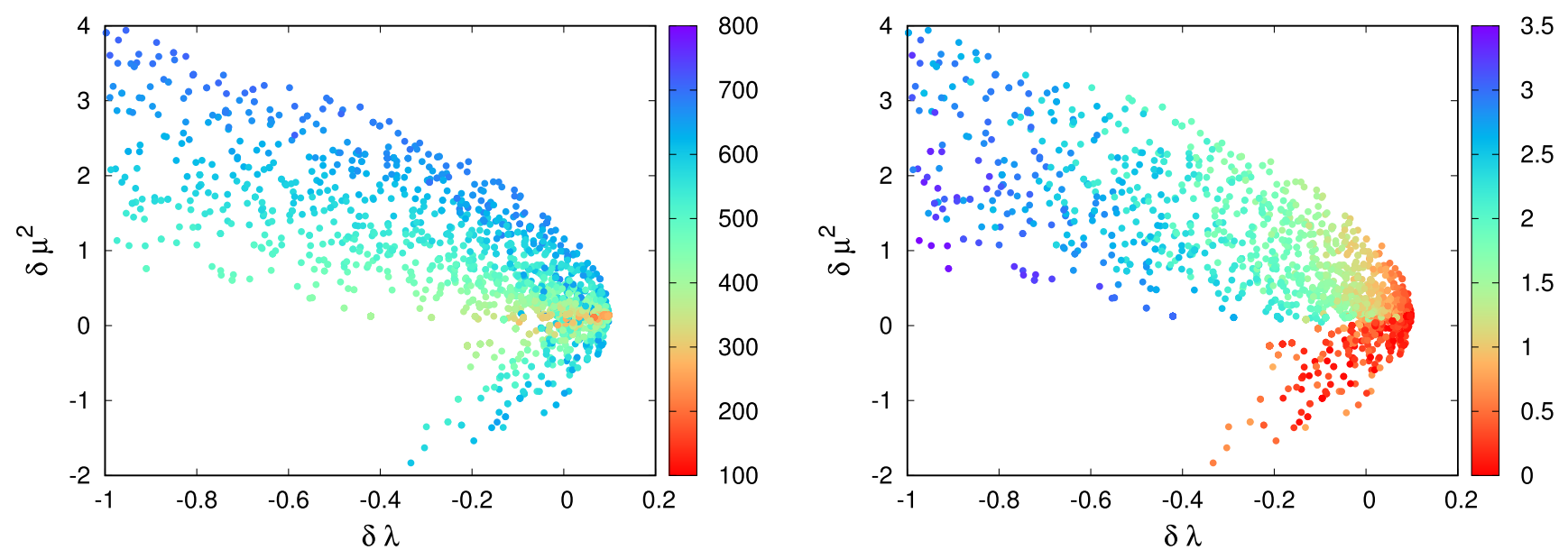

FIG. 3. The ratios $\delta \lambda$ and $\delta \mu^{2}$ that represent the relative corrections due to the inert members. In the palette, we read the large inert members mass (right) and its coupling to the Higgs, i.e., $\left|\lambda_{3}\right|\left(\left|\lambda_{3}+\lambda_{4} / 2 \pm \lambda_{5} / 2\right|\right)$ if $m_{H^{+}}>m_{H^{0}, A^{0}}\left(m_{H^{+}}<m_{H^{0}, A^{0}}\right)$.

on the observables $X=\lambda, \mu^{2}$, by showing the ratio $\delta X=$ $\left(X-X_{\text {tree-level }}\right) / X_{\text {tree-level }}$ in Fig. 3, where we consider 3000 benchmark points that fulfill all the conditions mentioned in the previous section.

One has to notice that the one-loop effect is very important for massive and strongly coupled inert

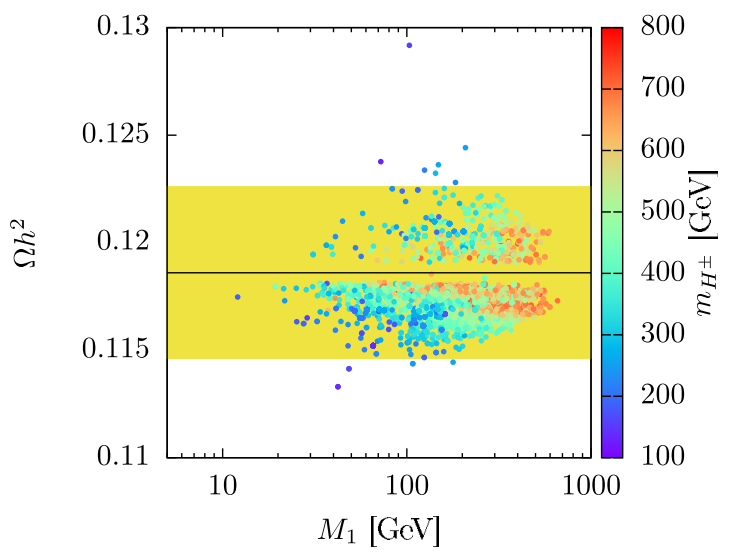

members. For instance, the inert corrections in (5) gives $\delta \lambda=-1 \sim 0.098$ and $\delta \mu^{2}=-1.83-3.94$, i.e., the Higgs mass and quartic coupling could be fully radiative $\delta \lambda \sim-1$.

In Fig. 4, we depict the DM relic density, coannihilation effect and the direct detection spin-independent cross
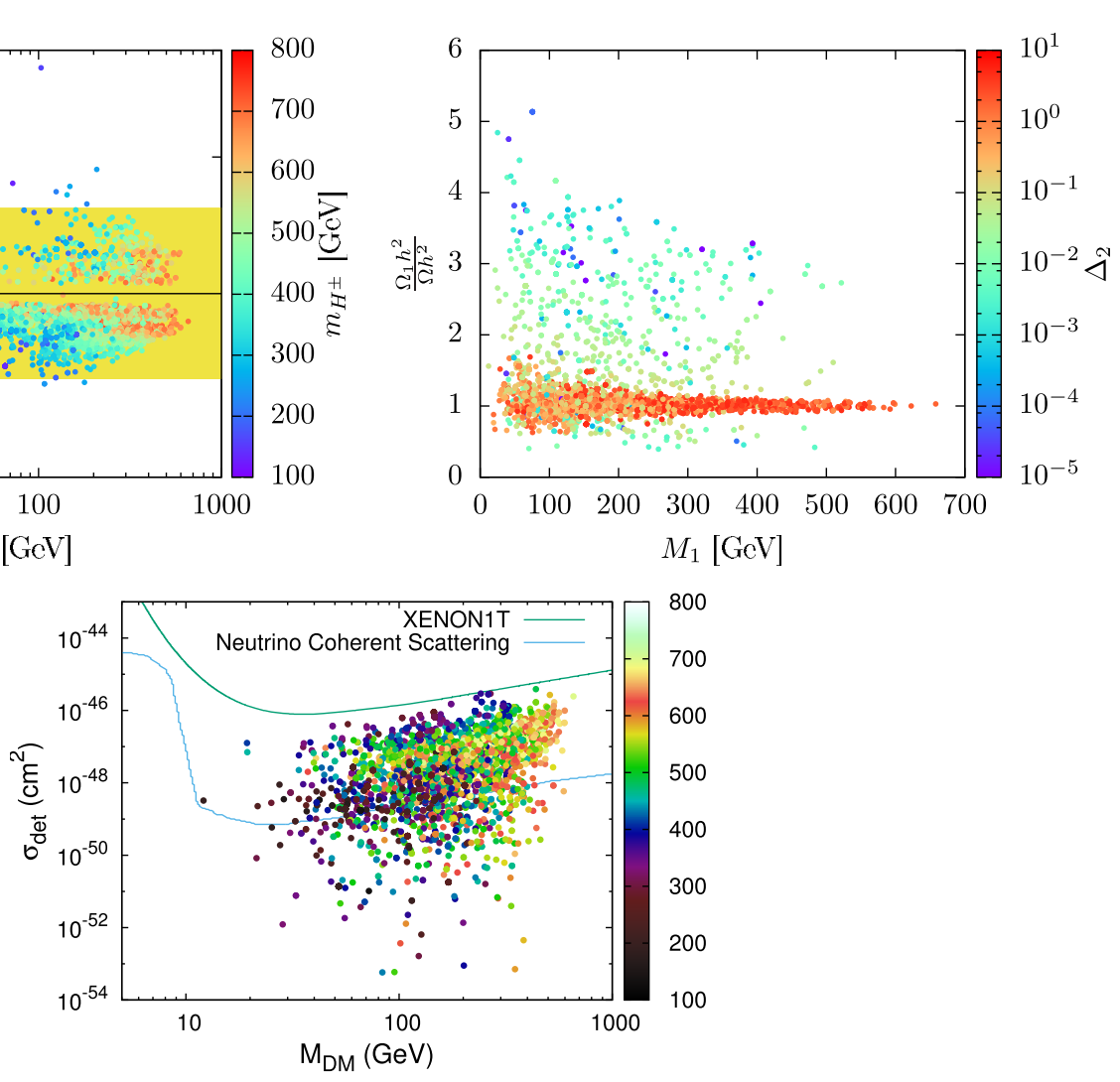

FIG. 4. Top left: the relic density of the DM as a function of its mass. The yellow band shows the experimental measurement of $\Omega_{N_{1}} h^{2}$ at $2 \sigma$. Top right: the ratio of $\Omega_{1} h^{2} / \Omega h^{2}$, where $\Omega_{1} h^{2}\left(\Omega h^{2}\right)$ does (not) include the coannihilation effect. The palette represents the degeneracy parameter $\Delta_{2}$ defined in (24). Bottom: the direct detection cross section as a function of the DM mass, with the exclusion plot obtained from the Xenon1T experiment. The blue curve represents the irreducible neutrino background due to the coherent neutrino-nucleus scattering. 

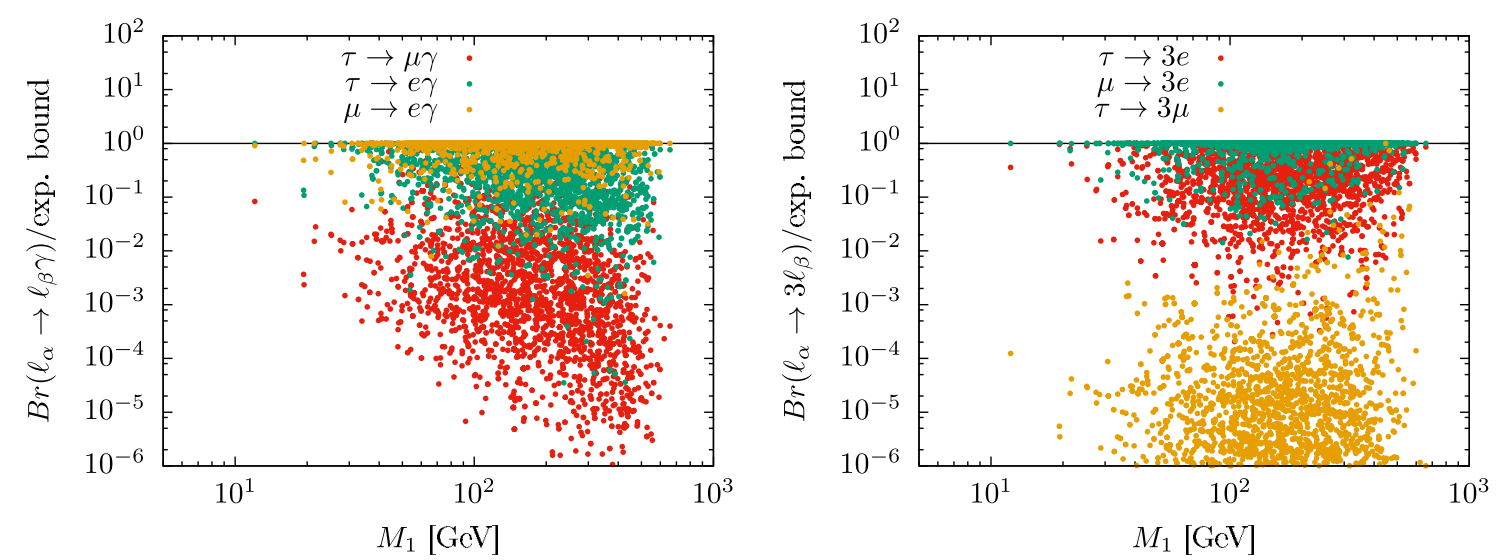

FIG. 5. The branching ratios $\operatorname{Br}\left(\ell_{\alpha} \rightarrow \ell_{\beta}+\gamma\right)$ (left) and $\operatorname{Br}\left(\ell_{\alpha} \rightarrow 3 \ell_{\beta}\right)$ (right) normalized by their experimental bounds as a function of $M_{1}$.

section as a function of the DM mass for the benchmark points used in Fig. 3.

From Fig. 4, one remarks that: (1) most of the benchmark points are within the $1 \sigma$ envelope on the relic density measurement. (2) We see that the lightest RHN can be a viable DM candidate for masses in the range $10-700 \mathrm{GeV}$ and with a spin-independent direct detection cross section below the experimental bound. Note that a large fraction of the benchmark points are above the irreducible neutrino background and hence can be probed in future direct detection experiments. (3) The ratio coannihilation effect that is presented with the ratio $\Omega_{1} h^{2} / \Omega h^{2}$, where $\Omega_{1} h^{2}$ $\left(\Omega h^{2}\right)$ refers to the relic density within (without) the coannihilation effect. As expected, the coannihilation effect is important only for benchmark points where the two RHN's $\left(N_{1}, N_{2}\right)$ are degenerate, i.e., very small mass difference values. It is worth mentioning that even though one could allow $M_{1}$ to vary over the mass range in (27), for DM heavier than $700 \mathrm{GeV}$ the relic density requires the couplings $h_{i, 1}$ to be much larger than unity so that the relic density will be in agreement with the observed value.

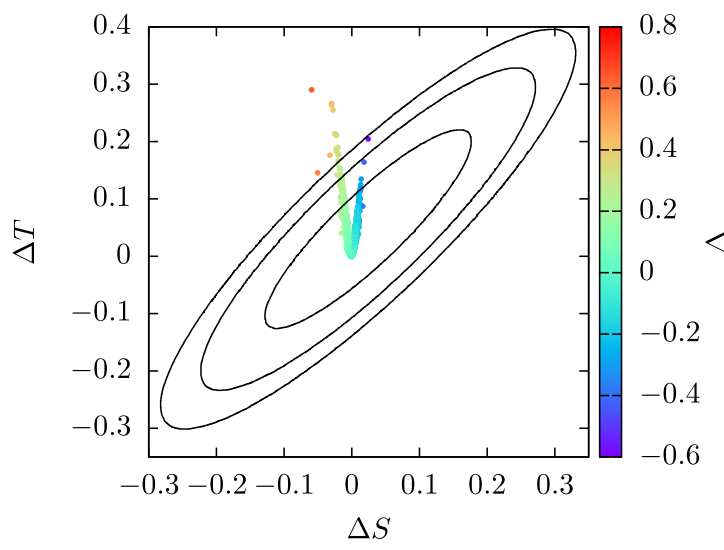

However, this will make the constraints on the LFV processes very difficult to satisfy.

For the same benchmark points, we present in Fig. 5, the different branching fractions of the LFV processes $\ell_{\alpha} \rightarrow$ $\ell_{\beta}+\gamma$ (left panel) and $\ell_{\alpha} \rightarrow 3 \ell_{\beta}$ (right panel), normalized to their experimental bounds, versus the DM mass. We Note that the stringent lepton flavor violation constraint comes from the process $\mu \rightarrow e \gamma$, where it is severely fulfilled for most of the benchmark points. This could be achieved by cancellation between different terms in (16), since the right relic density value does not allow the Yukawa couplings to have smaller values.

In Fig. 6-left, we depict the oblique parameters $S$ versus $T$ where the different ellipses represent the 68\%, 95\%, and 99\% CL intervals obtained from the precise measurements of various observables. The color map shows a ratio defined by

$$
\Delta=\frac{2 m_{H^{ \pm}}-m_{A^{0}}-m_{H^{0}}}{2 m_{H^{ \pm}}}
$$

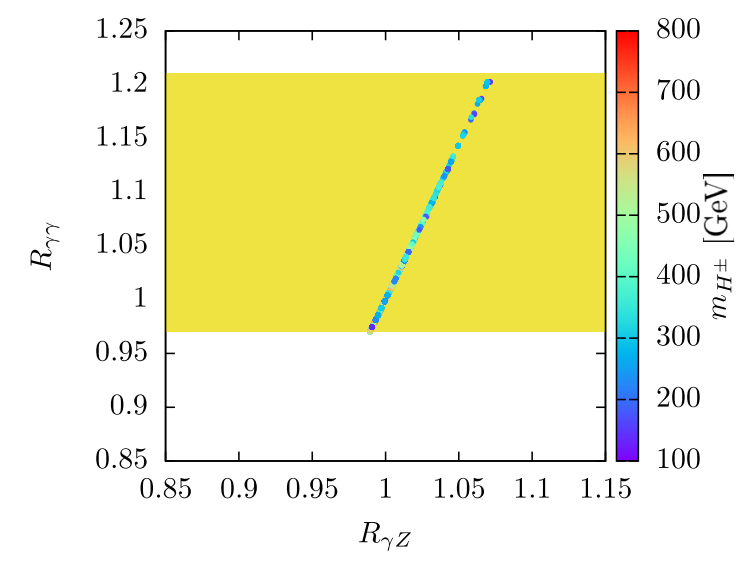

FIG. 6. Left: Constraints due to oblique parameters $S$ and $T$ with the palette showing the ratio $\Delta$ defined in (28). Right: the scatter plot of $\left(R_{\gamma \gamma}, R_{\gamma Z}\right)$, where the palette shows the value of $m_{H^{ \pm}}$allowed by theoretical and experimental constraints. The yellow band in the right panel shows the $1 \sigma$ interval of $R_{\gamma \gamma}$ of both ATLAS and CMS. 

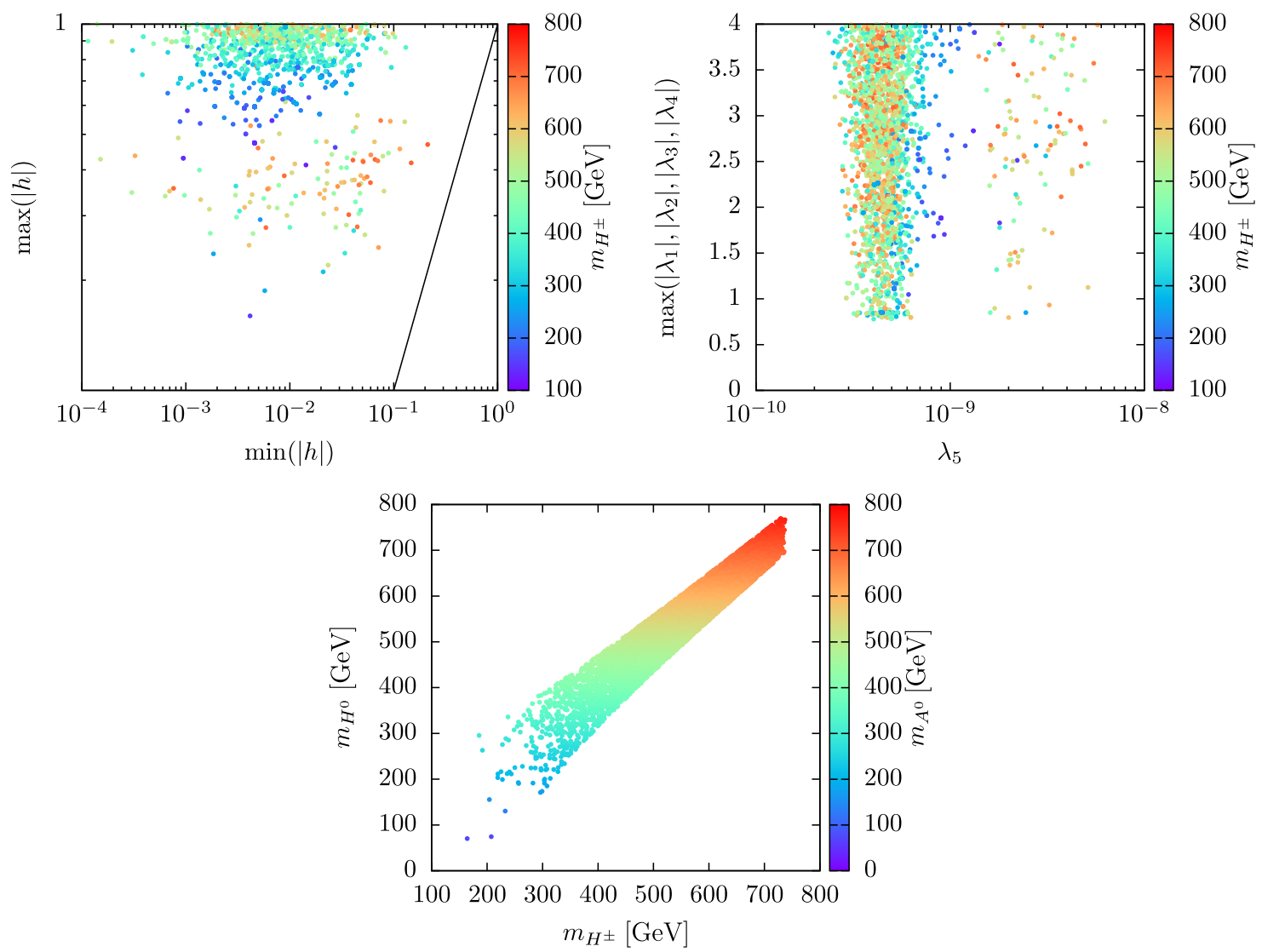

FIG. 7. Allowed model parameters by various constraints. Top left: $\max (|h|)$ versus $\min (|h|)$ where $h \equiv h_{i j}$ are the new Yukawa couplings (2). Top right: $\max \left(\lambda_{1}, \lambda_{2},\left|\lambda_{3}\right|,\left|\lambda_{4}\right|\right)$ versus $\lambda_{5}$. Bottom panel: scatter plot of $m_{H^{ \pm}}$versus $m_{H^{0}}$, where $m_{A^{0}}$ is shown in the palette.

which parametrizes how close is the charged Higgs mass to the arithmetic mean of the $C P$-even and $C P$-odd Higgs masses. In the right panel of Fig. 6, we show $R_{\gamma \gamma}$ vs $R_{\gamma Z}$. As can be seen from left panel in the figure, one remarks that the electroweak precision tests exclude the benchmark points with larger values of the parameter $\Delta$. From Fig. 6-right, one can see that the two branching ratios are proportional to each other due to the nature of the new additional scalar multiplet, i.e., the inert doublet. This can be understood from the factor $\left(1-2 s_{\mathrm{w}}^{2}\right) / c_{\mathrm{w}}$ in (19).

We give in Fig. 7 the range of parameter space of the model after passing all the theoretical and experimental constraints, leading to the results presented in the previous figures. In the left top panel, we show $\min (|h|)$ versus $\max (|h|)$ where $h \equiv h_{i j}$ are the new Yukawa couplings. The line displayed in that figure define the regime couplings are degenerate couplings regime, and farther from it the less degenerate the couplings are. Moreover, the Yukawa h-couplings values that fit the neutrino oscillation, fulfill the LFV and the relic density have the same order of magnitude whether the $C P$ violating phases are vanishing or not. Thus, in this model there is no favored values or range of $C P$ violating phases. Furthermore, in both cases of normal and inverted neutrino mass hierarchy, the allowed parameter space is similar. For the case of real Yukawa hcouplings, they need to be about one order of magnitude smaller than the complex case of complex, which is due to the fact that the cancellations in (16) required to fulfill the LFV constraints is much easier to achieve for complex valued Yukawa couplings than real ones.

In the right top panel of the same figure, we show $\max \left(\left|\lambda_{1,2,3,4}\right|\right)$ as a function of the coupling $\left|\lambda_{5}\right|$. One can see that $\lambda_{5}$ take very small values, and this is required to be in agreement with the observed neutrino masses and mixing angles. This allow the new Yukawa couplings $h_{i j}$ to be unsuppressed as opposed to the case considered in [22]. The bottom panel, represents the allowed masses of the $C P$-odd scalar, $m_{A^{0}}$, projected on the $\left(m_{H^{0}}, m_{H^{ \pm}}\right)$plan. The implication of the smallness of $\lambda_{5}$ is that the $C P$-odd and $C P$-even states are quasidegenerate, which render the constraints on the oblique parameters easy to satisfy.

\section{COLLIDER PHENOMENOLOGY}

\section{A. Possible signatures}

The RHNs, $N_{i}$, can be pair produced through several processes both at hadron as well at lepton colliders. At hadron colliders, however, it cannot be produced directly 

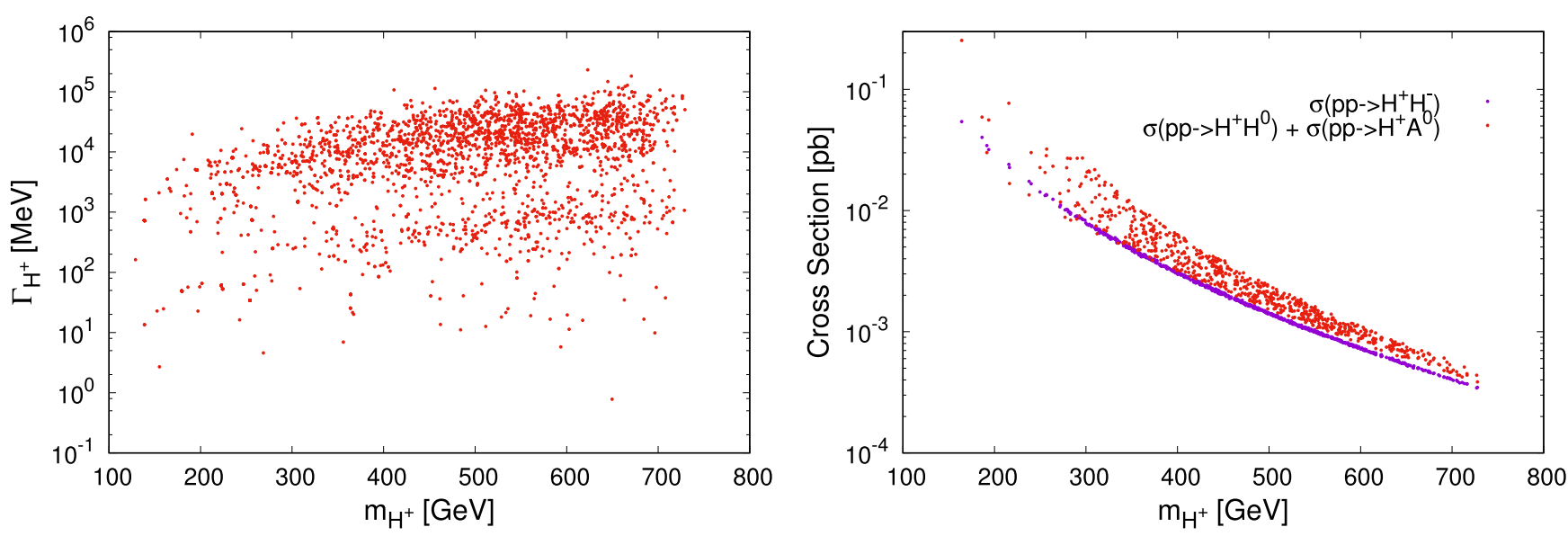

FIG. 8. Total width of the charged Higgs boson (left panel) and its inclusive cross section (right panel) as a function of $m_{H^{ \pm}}$.

because of the absence of the vertices $Z^{0} N \bar{N}, \gamma N \bar{N}$ and $h N \bar{N}$ in this model, and can only be found in the decay products of the inert doublet members. At lepton colliders such as the ILC or the FCC-ee, $N_{i}$ can be produced in $t$-channel processes with charged boson exchange. For the lightest RHN, $N_{1}$, when produced at colliders, it is accounted as missing energy, however, $N_{2}$ and $N_{3}$ depending on their decay width $\Gamma\left(N_{2,3} \rightarrow N_{1}+\ell^{+} \ell^{-}\right)$may or may not behave as missing energy. This depends on the decay lengths $l_{i}=E_{i} / M_{i} / \Gamma_{N_{i}}(i=2,3)$, with $E_{i}$ and $\Gamma_{N_{i}}$ are the energy and total decay width of $N_{i}$, respectively.

From the different theoretical and experimental constraints, especially from neutrino mass and the electroweak precision observables, the neutral scalar particles are degenerate and hence the decays such as $A^{0} \rightarrow H^{0} Z^{0}$ are kinematically forbidden. Thus, these particles can only undergo invisible decays (if kinematically allowed) into a RHN $N_{i}$ and a SM neutrino. At the LHC, the charged Higgs boson can be produced in pair or in association with $A^{0} / H^{0}$ with $\sigma \simeq 200 \mathrm{fb}$ for light charged Higgs boson mass in the $H^{0} H^{ \pm}\left(A^{0} H^{ \pm}\right)$mode (see Fig. 8). This channel, for $\Delta_{H^{ \pm}}=m_{H^{ \pm}}-m_{H^{0}, A^{0}}<M_{W^{ \pm}}$, leads exclusively to the spectacular mono-lepton signature. While dilepton signals can be observed in the case of the pair production of charged Higgs boson again for $\Delta_{H^{ \pm}}<M_{W^{ \pm}}$. On the other hand, for $\Delta_{H^{ \pm}}>M_{W^{ \pm}}$, the decays $H^{ \pm} \rightarrow A^{0} / H^{0} W^{ \pm}$are kinematically allowed and hence a wide range of signatures are possible (see Table I). For the case of $H^{0} H^{0}\left(A^{0} A^{0}\right.$ or $H^{0} A^{0}$ ) channels, the two particles decay exclusively into a $N_{i} \nu_{\ell}$ giving a mono-jet or mono-photon signature where the additional jet/photon is produced from initial state radiation (ISR) off the scattered quarks. However, due to the large $\alpha_{s}(Q) / \alpha_{\mathrm{em}}(Q)$ ratio, QCD radiation is dominant and monojet signature is more relevant in this case.

At lepton colliders, the $N_{1}$ can be pair produced through $H^{ \pm}$exchange leading to monophoton signature where the additional photon is emitted either from the $e^{ \pm}$lines or from the intermediate charged Higgs boson. Pair production of charged Higgs boson or a $C P$-odd ( $C P$-even) particle is also possible at lepton colliders. In Table I, we summarize the different signatures that can be used to either look for $N_{1}$ in the next LHC-run or to constrain the model using the old measurements.

In Fig. 8, we show the total decay width of the charged Higgs (left) as a function of its mass for some of the benchmarks used previously. We see that the width can be

TABLE I. Summary of various signatures of the inert Higgs doublet members and the lightest RHN at the LHC and lepton colliders.

\begin{tabular}{|c|c|c|}
\hline Process & Decay mode & Signature \\
\hline$p p, e^{+} e^{-} \rightarrow H^{ \pm} H^{\mp}$ & $\begin{array}{c}H^{ \pm} \rightarrow W^{ \pm} H^{0} / A^{0} \rightarrow q_{1} \bar{q}_{2} N_{1} \nu_{\ell}, H^{\mp} \rightarrow W^{\mp} H^{0} / A^{0} \rightarrow q_{3} \bar{q}_{4} N_{1} \bar{\nu}_{\ell} \\
H^{ \pm} \rightarrow W^{ \pm} H^{0} / A^{0} \rightarrow \ell^{ \pm} \nu_{\ell} N_{1} \nu_{\ell}, H^{\mp} \rightarrow W^{\mp} H^{0} / A^{0} \rightarrow q_{3} \bar{q}_{4} N_{1} \bar{\nu}_{\ell} \\
H^{ \pm} \rightarrow W^{ \pm} H^{0} / A^{0} \rightarrow \ell_{1}^{ \pm} \nu_{\ell} N_{1} \nu_{\ell}, H^{\mp} \rightarrow W^{\mp} H^{0} / A^{0} \rightarrow \ell_{2}^{\mp} \bar{\nu}_{\ell} N_{1} \nu_{\ell} \\
H^{ \pm} \rightarrow W^{ \pm} H^{0} / A^{0} \rightarrow \ell_{1}^{ \pm} \nu_{\ell} N_{1} \nu_{\ell}, H^{\mp} \rightarrow \ell_{2}^{\mp} N_{1} \\
H^{ \pm} \rightarrow W^{ \pm} H^{0} / A^{0} \rightarrow q_{1} \bar{q}_{2} N_{1} \nu_{\ell}, H^{\mp} \rightarrow \ell^{\mp} N_{1}\end{array}$ & $\begin{array}{c}4 \text { jets }+\not E_{T} \\
1 \ell+2 \text { jets }+\not E_{T} \\
2 \ell+\not E_{T} \\
2 \ell+\not E_{T} \\
1 \ell+2 \text { jets }+\not E_{T}\end{array}$ \\
\hline$p p, e^{+} e^{-} \rightarrow H^{ \pm} H^{0} / A^{0}$ & $\begin{array}{c}H^{ \pm} \rightarrow W^{ \pm} H^{0} / A^{0} \rightarrow q_{1} \bar{q}_{2} N_{1} \nu_{\ell}, H^{0} \rightarrow N_{1} \nu_{\ell} \\
H^{ \pm} \rightarrow W^{ \pm} H^{0} / A^{0} \rightarrow \ell^{ \pm} \nu_{\ell} N_{1} \nu_{\ell}, H^{0} \rightarrow N_{1} \nu_{\ell} \\
H^{ \pm} \rightarrow N_{1} \ell^{ \pm}\end{array}$ & $\begin{array}{c}1 \ell+2 \text { jets }+\mathscr{E}_{T} \\
1 \ell+E_{T} \\
1 \ell+E_{T}\end{array}$ \\
\hline$p p, e^{+} e^{-} \rightarrow H^{0} H^{0}$ & $H^{0} \rightarrow N_{1} \nu_{\ell}$ & $\mathscr{E}_{T}+$ ISR (mono-jet, mono- $\gamma$ ) \\
\hline$e^{+} e^{-} \rightarrow N_{1} N_{1} \gamma$ & stable final state & $E_{T}+\gamma$ \\
\hline
\end{tabular}




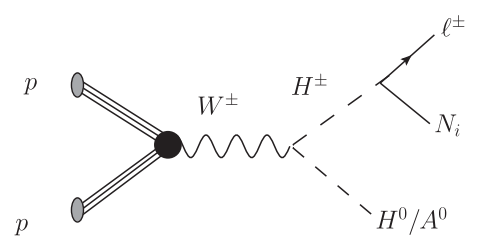

(a)

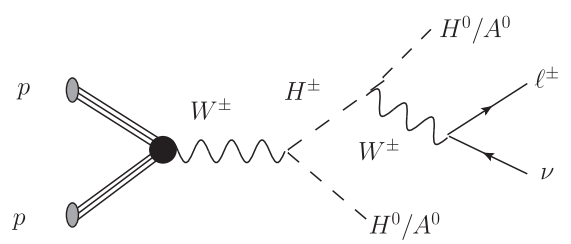

(b)

FIG. 9. Feynman diagrams for the charged monolepton signature.

as large as few hundred of $\mathrm{GeV}$ for heavy charged Higgs bosons. The reason is that scalar decays $H^{ \pm} \rightarrow W^{ \pm} A^{0} / H^{0}$ are kinematically allowed in certain regions of the parameter space. In the right panel, we present the cross section at the LHC at $\sqrt{s}=14 \mathrm{TeV}$ of $H^{ \pm} H^{\mp}$ and $H^{ \pm} H^{0}\left(A^{0}\right)$ as a function of the charged Higgs mass. We can see that the $H^{ \pm} H^{0}\left(A^{0}\right)$ have larger cross sections and approach $200 \mathrm{fb}$ for light charged Higgs boson. We stress that for heavy scalar masses, the production cross section is extremely small. This makes the observation of the new states extremely difficult at moderate luminosities.

In the rest of this section, we will investigate the monolepton signature at the LHC, that is mentioned in Table I.

\section{B. Monolepton signature at the LHC}

As a benchmark study, we consider the $1 \ell+E_{T}^{\text {miss }}$ $(\ell=e, \mu)$ final state in $p p$ collisions at $\sqrt{s}=14 \mathrm{TeV}$ and a luminosity of $300 \mathrm{fb}^{-1}$. Such a signature can arise from $H^{ \pm} H^{0} / A^{0}$ production followed by the decay of the charged Higgs either into (1) a charged lepton and a Majorana fermion or (2) a $W$ gauge boson and a dark Higgs where the gauge boson decays leptonically. Feynman diagrams showing the $1 \ell+E_{T}^{\text {miss }}$ in the signal process are depicted in Fig. 9.

Two benchmark points, denoted by $\mathrm{BP} 1$ and $\mathrm{BP} 2$, are considered in this study where the corresponding parameters are given in Table II. Moreover, the chosen benchmarks satisfy all the theoretical and experimental constraints and yield a cross section of order $460 \mathrm{fb}$ and $127 \mathrm{fb}$ for $\mathrm{BP} 1$ and $\mathrm{BP} 2$, respectively, as shown in Table III. A contribution from $H^{0} H^{0} W^{ \pm}, A^{0} A^{0} W^{ \pm}$with the $W$-boson decaying leptonically is relevant in BP1 since $\sigma\left(p p \rightarrow H^{0} H^{0} W^{ \pm} \rightarrow \ell^{ \pm} E_{T}^{\text {miss }}\right) \approx 45 \mathrm{fb}$. However, the same contribution is very negligible for BP2 and will

TABLE II. The parameters values the benchmark points BP1 and BP2.

\begin{tabular}{|c|c|c|c|c|}
\hline Benchmark Point & & \multicolumn{3}{|c|}{ Parameters } \\
\hline \multirow[t]{2}{*}{$\mathrm{BP} 1$} & \multirow{2}{*}{$\begin{array}{c}m_{N_{i}}(\mathrm{GeV}) \\
m_{H^{ \pm}}, m_{H^{0}}(\mathrm{GeV}) \\
\lambda_{2}, \lambda_{3}, \lambda_{4}, \lambda_{5} \\
h_{i j} / 10^{-2}\end{array}$} & \multicolumn{3}{|c|}{$\begin{array}{c}28.52,30.97,109 \\
164.2,70.49 \\
2.662,-0.153,-1.451,1.379 \times 10^{-10}\end{array}$} \\
\hline & & $\begin{array}{c}-14.218-i 0.997 \\
69.991+i 0.438 \\
-57.401+i 0.980\end{array}$ & $\begin{array}{l}-2.030-i 0.999 \\
-0.544+i 0.107 \\
-0.188+i 0.472\end{array}$ & $\begin{array}{c}62.265-i 0.025 \\
-5.559+i 0.719 \\
-24.365-i 0.419\end{array}$ \\
\hline \multirow[t]{2}{*}{ BP2 } & $\begin{array}{c}m_{N_{i}}(\mathrm{GeV}) \\
m_{H^{ \pm}}, m_{H^{0}}(\mathrm{GeV}) \\
\lambda_{2}, \lambda_{3}, \lambda_{4}, \lambda_{5}\end{array}$ & \multicolumn{3}{|c|}{$\begin{array}{c}17.93,29.27,54.49 \\
215.7,124 \\
1.069,0.429,-2.05,1.85 \times 10^{-10}\end{array}$} \\
\hline & $h_{i j} / 10^{-2}$ & $\left(\begin{array}{c}133.45+i 5.739 \\
0.79-i 1.028 \\
-68.17+i 2.869\end{array}\right.$ & $\begin{array}{c}0.239+i 0.071 \\
-2.867+i 2.957 \\
0.413+i 0.082\end{array}$ & $\left.\begin{array}{c}-26.05+i 0.57 \\
78.53-i 0.459 \\
-57.06-i 1.276\end{array}\right)$ \\
\hline
\end{tabular}

TABLE III. $\quad H^{ \pm} S\left(S=A^{0}, H^{0}\right)$ production cross section and decays branching ratios of the charged Higgs boson in the two benchmark scenarios.

\begin{tabular}{cccccc}
\hline \hline & $\sigma\left(p p \rightarrow H^{ \pm} H^{0} / A^{0}\right)$ & $\operatorname{Br}\left(W^{ \pm} H^{0} / A^{0}\right)$ & $\sum_{i} \operatorname{Br}\left(e^{ \pm} N_{i}\right)$ & $\sum_{i} \operatorname{Br}\left(\mu^{ \pm} N_{i}\right)$ & $\sum_{i} \operatorname{Br}\left(\tau^{ \pm} N_{i}\right)$ \\
\hline BP1 & $460.78 \mathrm{fb}$ & $37.22 \%$ & $19.74 \%$ & $24.07 \%$ & $18.95 \%$ \\
BP2 & $125.67 \mathrm{fb}$ & $14.46 \%$ & $49.62 \%$ & $16.25 \%$ & $21.02 \%$ \\
\hline \hline
\end{tabular}


not be taken into account. Furthermore, they have distinct features regarding collider phenomenology and dark matter relic density. For the first benchmark point (BP1), coannihilation effects are important since $\Delta_{2} \approx 0.08$, whereas, due to the relatively larger value of $\Delta_{2}$, co-annihilation effects are negligible for the second benchmark (BP2). However, in both scenarios, the decay $H^{ \pm} \rightarrow W^{ \pm} A^{0} / H^{0} \rightarrow$ $\ell^{ \pm} E_{T}^{\text {miss }}$ has a very small branching ratio, compared to $\operatorname{Br}\left(H^{ \pm} \rightarrow \ell^{ \pm} N_{i}\right)$, which is about $8.27 \%$ (3.21\%) for $\mathrm{BP} 1$ (BP2).

The background contributions to $\ell+E_{T}^{\text {miss }}$ signal can be classified into two categories: irreducible and reducible. The $W$ production followed by its leptonic is the dominant irreducible background with a cross section of order $\mathcal{O}(20) \mathrm{nb}$ at leading order (LO). Diboson processes, $W Z, W W$, and $Z Z$, contribute as well to the background sources in the $\ell+E_{T}^{\text {miss }}$ final state especially $W(\rightarrow \ell \nu) \times$ $Z(\rightarrow \nu \bar{\nu})$ which is irreducible. Important contributions might come from $t \bar{t}$ and single top production where the top quark decays leptonically. Furthermore, there are several background processes whose contribution cannot be estimated at the parton level. In such background categories, charged lepton and missing energy arise either from (i) multijet production where they are produced from hadron decays, and (ii) in Drell-Yan process $\left(Z / \gamma^{*} \rightarrow\right.$ $\ell^{+} \ell^{-}$) where one lepton is not detected. We do not consider these background in this study since their contribution can be significantly reduced by imposing isolation cuts and by requiring that the lepton and missing must have a back-toback topology. These requirements are translated into cuts on the ratio of $p_{T}^{\ell} / E_{T}^{\text {miss }}$ and $\Delta \Phi\left(\ell, E_{T}^{\text {miss }}\right)$. A perfect balance in the transverse plane between the charged lepton is reached when a charged lepton is produced in association with an invisible particle yielding a ratio $p_{T}^{\ell} / E_{T}^{\text {miss }} \simeq 1$ and an azimuthal separation $\Delta \Phi \simeq \pi$. However, in hadronic collisions, there are QCD radiation off partons in the proton beams which result in a recoil of the lepton and missing energy system and yield a shift in the values $p_{T}^{\ell} / E_{T}^{\text {miss }}$ and $\Delta \Phi\left(\ell, E_{T}^{\text {miss }}\right)$ but with an unmodified peak position. For reference, in Fig. 10 we show the imbalance between the lepton $p_{T}^{\ell}$ and transverse missing energy in the two benchmark points and the SM background. We can see that for the signal, $W^{ \pm}$and $W^{ \pm} Z$, this distribution is peaked around a value about 1 while it is a smooth function in the case of top quark processes.

Signal events were generated at LO using CALCHEP [46] while $W^{ \pm}$and $W^{ \pm} Z$ events were generated using MADGRAPH5_AMC@NLO [47]. PYTHIA8 [48] was used for showering and hadronization of the parton level events and for generation of top quark events. Due to its large cross section, $W^{ \pm}$boson events were generated with strong cuts, i.e., with $p_{T}^{\ell}>100 \mathrm{GeV}$ and $E_{T}^{\text {miss }}>50 \mathrm{GeV}$. Such cuts reduce the total $W$ boson production cross section from $19 \mathrm{nb}$ to about $6 \mathrm{pb}$. While for the other background processes, no cuts were imposed on the generated MC samples. At the analysis level, further cuts are imposed on the charged

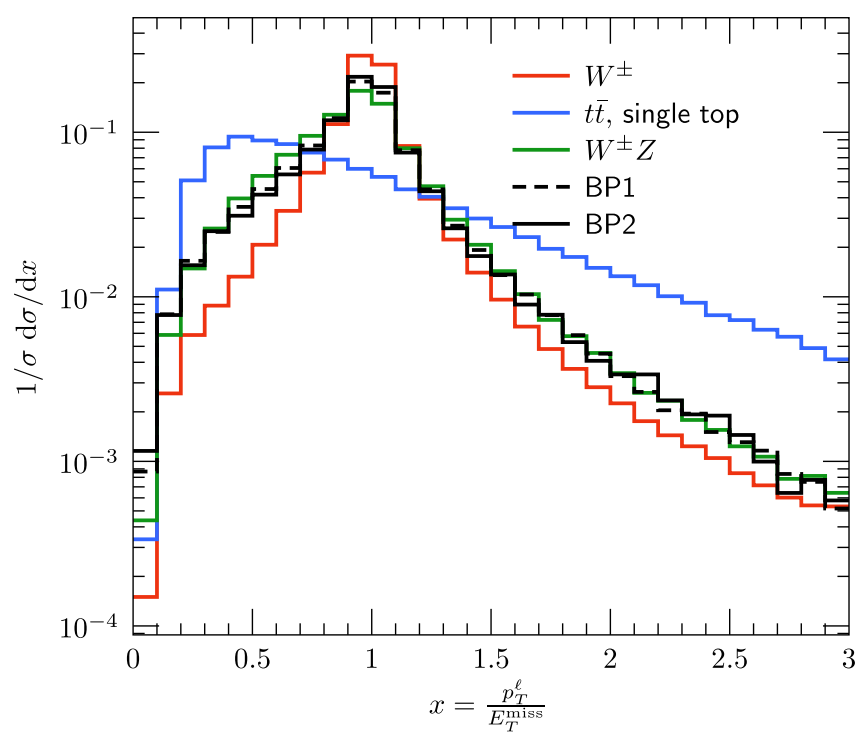

FIG. 10. Transverse momentum imbalance between the charged lepton and the missing energy in the two benchmark points and the SM background. The distribution is normalized to unity.

lepton $p_{T}^{\ell}$ and $E_{T}^{\text {miss }}$ to further reduce the SM background contribution. We, first, select events which contains exactly one charged lepton (either electron or muon) with $p_{T}^{\ell}>$ $30 \mathrm{GeV}$ and $|\eta|<2.4$, and a missing transverse energy (MET) with $E_{T}^{\text {miss }}>30 \mathrm{GeV}$. Furthermore, the imbalance between the lepton $p_{T}$ and MET was required to be $0.4<p_{T}^{\ell} / E_{T}^{\text {miss }}<1.4$. Such a cut reduce the amount of events for the signal and the irreducible background by about 20\%-25\%. However, reducible backgrounds such as $t \bar{t}$ and single top quark production are reduced by a factor of 0.91 . No cuts were imposed on the jet multiplicity, jet $p_{T}$ or $b$-tagging since jet activity is involved in all the processes. We recommend for a more complete analysis to be done at the experimental level. We further refine our selection criteria and define two signal regions; the first signal region is defined by $p_{T}^{\ell}>250 \mathrm{GeV}$ and $E_{T}^{\text {miss }}>150 \mathrm{GeV}$ while the second signal region is defined as $p_{T}^{\ell}>250 \mathrm{GeV}$ and $E_{T}^{\text {miss }}>300 \mathrm{GeV}$. In Fig. 11, we show the corresponding distributions for the signal as well as background events.

To quantify the potential discovery of the model in the two benchmark points, we use the general formula for the signal significance which is defined by

$\mathcal{S}=\sqrt{2 \times\left[\left(N_{\mathrm{S}}+N_{\mathrm{BG}}\right) \times \log \left(1+\frac{N_{\mathrm{S}}}{N_{\mathrm{BG}}}\right)-N_{\mathrm{S}}\right]}$,

We compute $\mathcal{S}$ for different values of the cut on the transverse mass of the lepton and missing transverse energy system defined by

$$
M_{T}=\sqrt{2 p_{T}^{\ell} E_{T}^{\text {miss }}\left(1-\cos \Delta \Phi\left(\ell, E_{T}^{\text {miss }}\right)\right)}
$$



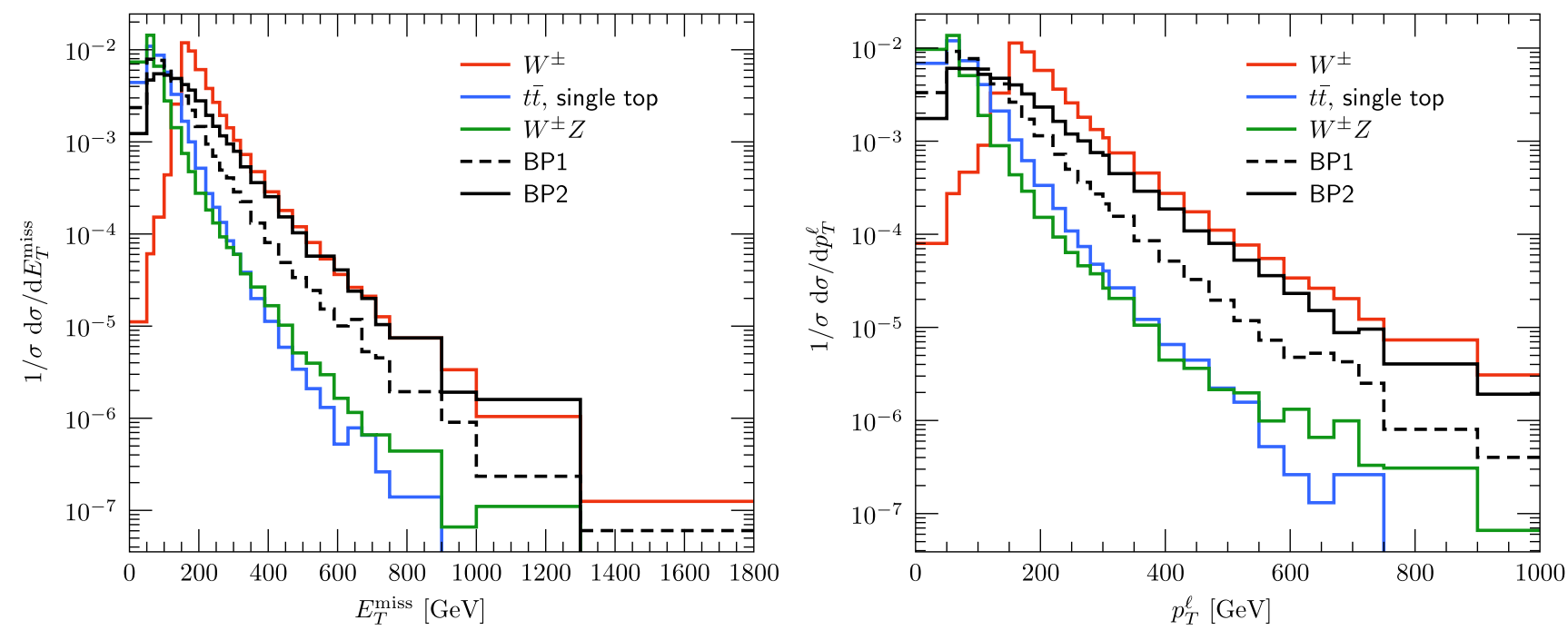

FIG. 11. Normalized distributions for missing transverse energy (left panel) and lepton $p_{T}$ (right panel) for signal and background events.
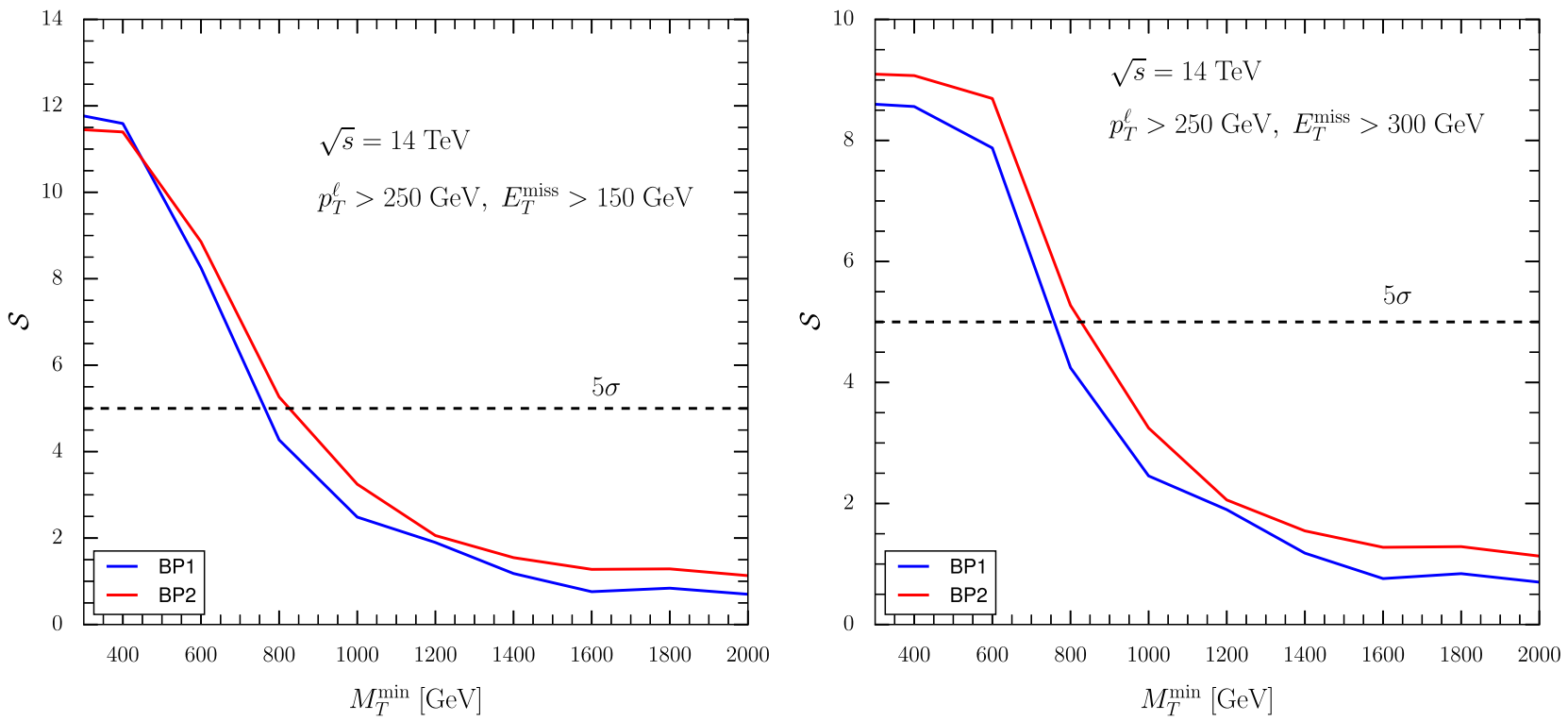

FIG. 12. Signal significance as function of $M_{T}^{\min }$ for the first (left panel) and second (right panel) signal regions.

In Fig. 12, we plot the significance as function of the cut on $M_{T}$ for the two considered benchmark points and in the two signal regions. For $M_{T}^{\min }<1 \mathrm{TeV}$, the significance reaches about $5 \sigma$ level, and decreases quickly for $M_{T}^{\min }>1 \mathrm{TeV}$. This behavior at $M_{T}^{\min }>1 \mathrm{TeV}$ is due to the relatively low statistics at that region which is a consequence of the light scalars chosen in our benchmark points. On the other hand, benchmark points with heavier scalars hardly achieve the $5 \sigma$ level due to the smallness of the corresponding cross section. We notice that in the observability region, systematic uncertainties are quite moderate. They are dominated by uncertainties due to electron and muon energy resolution. Statistical uncertainties are also not important in this region of interest. We found that even for lower luminosities of about $100 \mathrm{fb}^{-1}$, the significance can reach the $5 \sigma$ level for $M_{T}^{\min }<600 \mathrm{GeV}$ in BP1. We leave a more detailed study of all the systematic uncertainties in monolepton signatures and interplay with the other channels for a future work [49].

\section{CONCLUSION}

In this paper, we considered the inert Higgs doublet model extended by three right handed neutrinos where both neutrino mass and dark matter are addressed. We considered the DM to be the lightest right handed neutrino and showed that its relic density can be in agreement with the observation provided that Yukawa couplings in the neutrino sector are not highly suppressed. We carried out a detailed numerical analysis to determine the different regions of the parameter space that are consistent with theoretical and 
experimental constraints. Fitting the observed neutrino mass squared differences and mixing angles requires that the $C P$-odd and the $C P$-even components of the inert doublet to have quasidegenerate masses. We also discussed a number of experimental signatures of this model at high energy colliders. In particular, monolepton, monojet, and monophoton signals are particularly interesting and can be used to search for the right-handed neutrinos both at the LHC and future lepton colliders, such as the ILC. We have performed at detailed analysis of the monolepton signature at the LHC and showed that with a $300 \mathrm{fb}^{-1}$ luminosity it is possible to probe the right-handed neutrino signal.

\section{ACKNOWLEDGMENTS}

We want to thank M. Chekkal for his help in the results production in Fig. 8. The work of A. J. was supported by
Shanghai Pujiang Program and by the Moroccan Ministry of Higher Education and Scientific Research MESRSFC and CNRST: "Projet dans les domaines prioritaires de la recherche scientifique et du developpement technologique": PPR/2015/6.

\section{APPENDIX A: THE CROSS SECTION OF $N_{i}-N_{k}$ COANNIHILATION}

In this Appendix, we derive the analytic expression annihilation cross of two right handed neutrinos, $N_{i}$ and $N_{k}$, into charged leptons or light neutrinos. For the charged leptons channel, there are two diagrams that contribute to this process, as shown in Fig. 13.

After averaging and summing over the initial and final spin states, the corresponding squared amplitude is given by

$$
|\mathcal{M}|^{2}=\frac{\left|g_{i \alpha} g_{k \beta}^{*}\right|^{2}}{4}\left[\frac{\left(M_{i}^{2}+m_{\ell_{\alpha}}^{2}-t\right)\left(M_{k}^{2}+m_{\ell_{\beta}}^{2}-t\right)}{\left(t-m_{H^{+}}^{2}\right)^{2}}+\delta_{i k} \frac{\left(M_{i}^{2}+m_{\ell_{\alpha}}^{2}-u\right)\left(M_{i}^{2}+m_{\ell_{\beta}}^{2}-u\right)}{\left(u-m_{H^{+}}^{2}\right)^{2}}-\delta_{i k} \frac{2 M_{i}^{2}\left(s-m_{\ell_{\alpha}}^{2}-m_{\ell_{\beta}}^{2}\right)}{\left(t-m_{H^{+}}^{2}\right)\left(u-m_{H^{+}}^{2}\right)}\right],
$$

with $s, t, u$ and $s$ are the Lorentz invariant Mandelstam variables.

After integrating over the phase space, the cross section times the relative velocity of $N_{i}$ and $N_{k}$ reads

$$
\begin{aligned}
v_{r} \sigma= & \frac{\left|g_{i \alpha} g_{k \beta}^{*}\right|^{2}}{32 \pi} \frac{\sqrt{s^{2}-2 s\left(m_{\ell_{\alpha}}^{2}+m_{\ell_{\beta}}^{2}\right)+\left(m_{\ell_{\alpha}}^{2}-m_{\ell_{\beta}}^{2}\right)^{2}}}{s^{2}-\left(M_{i}^{2}-M_{k}^{2}\right)^{2}}\left[\frac{A_{1}^{2}-B^{2}+Q_{1} Q_{2}}{A_{1}^{2}-B^{2}}-\frac{Q_{1}+Q_{2}}{2 B} \log \left|\frac{A_{1}+B}{A_{1}-B}\right| \mid+\delta_{i k}\right. \\
& \left.\times\left\{\frac{A_{2}^{2}-B^{2}+Q_{1} Q_{2}}{A_{2}^{2}-B^{2}}-\frac{Q_{1}+Q_{2}}{2 B} \log \left|\frac{A_{2}+B}{A_{2}-B}\right|-\frac{M_{i}^{2}\left(s-m_{\ell_{\alpha}}^{2}-m_{\ell_{\beta}}^{2}\right)}{B\left(Q_{1}+Q_{2}-s\right)}\left(\log \left|\frac{A_{1}+B}{A_{1}-B}\right|+\log \left|\frac{A_{2}+B}{A_{2}-B}\right|\right)\right\}\right],
\end{aligned}
$$

where

$$
\begin{aligned}
& Q_{1}=M_{i}^{2}+m_{\ell_{\alpha}}^{2}-m_{H^{+}}^{2}, Q_{2}=M_{k}^{2}+m_{\ell_{\beta}}^{2}-m_{H^{+}}^{2}, \\
& A_{1}=\frac{M_{i}^{2}+M_{k}^{2}+m_{\ell_{\alpha}}^{2}+m_{\ell_{\beta}}^{2}-2 m_{H^{+}}^{2}-s}{2}-\frac{\left(M_{i}^{2}-M_{k}^{2}\right)\left(m_{\ell_{\alpha}}^{2}-m_{\ell_{\beta}}^{2}\right)}{2 s}, \\
& A_{2}=M_{i}^{2}-m_{H^{+}}^{2}+\frac{m_{\ell_{\alpha}}^{2}+m_{\ell_{\beta}}^{2}-s}{2}, B=2 \frac{\sqrt{s^{2}-2 s\left(m_{\ell_{\alpha}}^{2}+m_{\ell_{\beta}}^{2}\right)+\left(m_{\ell_{\alpha}}^{2}-m_{\ell_{\beta}}^{2}\right)^{2}}}{2 \sqrt{s}} p .
\end{aligned}
$$

Here $p=\sqrt{s^{2}-2 s\left(M_{i}^{2}+M_{k}^{2}\right)+\left(M_{i}^{2}-M_{k}^{2}\right)^{2}} / 2 \sqrt{s}$ is the magnitude of the momentum of the incoming RHN in the center of mass frame. The cross section of $\sigma\left(N_{i} N_{k} \rightarrow \nu_{\alpha} \nu_{\beta}\right)$ can be obtained by simply making the replacement $m_{H^{+}}^{2} \rightarrow \bar{m}^{2}=\frac{1}{2}\left(m_{H^{0}}^{2}+m_{A^{0}}^{2}\right)$ and $m_{\ell_{\alpha}}^{2}=m_{\ell_{\beta}}^{2}=0$, in the above expression (A2).

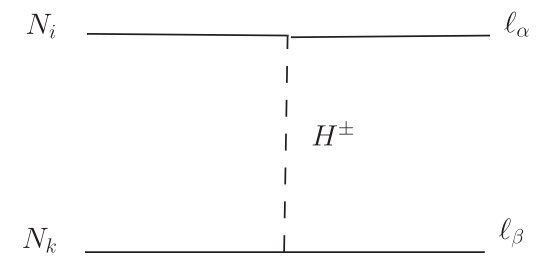

(a)

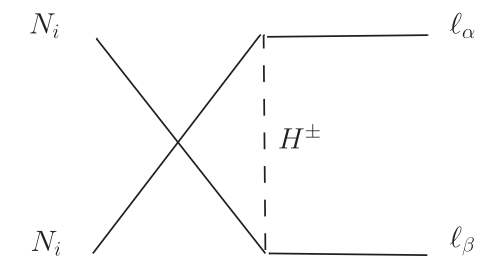

(b)

FIG. 13. Diagrams contributing to the annihilation $N_{i} N_{k} \rightarrow \ell_{\alpha}^{-} \ell_{\beta}^{+}$. Clearly, the second diagram is possible only if the two RHN's are identical $(i=k)$. Similar diagrams exit for the channels $N_{i} N_{k} \rightarrow \nu_{\alpha} \bar{\nu}_{\beta}$, where the mediators are $H^{0} / A^{0}$. 
In the nonrelativistic limit, we can expand the logarithms in (A2) in power of $p$, equivalently $B$, to obtain

$$
\begin{aligned}
v_{r} \sigma \approx & \frac{\left|g_{i \alpha} g_{k \beta}^{*}\right|^{2}}{32 \pi} \frac{\sqrt{s^{2}-2 s\left(m_{\ell_{\alpha}}^{2}+m_{\ell_{\beta}}^{2}\right)+\left(m_{\ell_{\alpha}}^{2}-m_{\ell_{\beta}}^{2}\right)^{2}}}{s^{2}-\left(M_{i}^{2}-M_{k}^{2}\right)^{2}} \\
& \times\left[\frac{A_{1}-Q-Q_{2}}{A_{1}}+\frac{Q_{1} Q_{2}}{A_{1}^{2}}\left(1+\frac{B^{2}}{A_{1}^{2}}\right)\right. \\
& +\delta_{i k}\left\{\frac{A_{2}-Q_{1}-Q_{2}}{A_{2}}+\frac{Q_{1} Q_{2}}{A_{2}^{2}}\left(1+\frac{B^{2}}{A_{2}^{2}}\right)\right. \\
& \left.\left.-\frac{2 M_{i}^{2}\left(s-m_{\ell_{\alpha}}^{2}-m_{\ell_{\beta}}^{2}\right)}{A_{2}^{2}}\right\}\right] .
\end{aligned}
$$

For $i=k=1$, the expression of the annihilation cross section agrees with the one given in [12].

\section{APPENDIX B: THE EFFECTIVE $h N_{1} \bar{N}_{1}$ COUPLING}

Here, we present the different contributions to the effective $h N_{1} \bar{N}_{1}$ coupling in terms of the PassarinoVeltman three-point functions (denoted by $C_{i}$ in what follows). To compute the effective $\tilde{y}_{h N_{1} \bar{N}_{1}}$ coupling, we consider the process $h(p) \rightarrow N_{1}\left(k_{1}\right)+\bar{N}_{1}\left(k_{2}\right)$, at oneloop level. The corresponding Feynman diagrams are depicted in Fig. 2. The evaluation of the Feynman amplitudes was performed analytically and compared to the result of FEYNARTS and FormCALC [50] while numerical evaluations of the coupling were performed with the help of the LoOpToOLS package [51] and compared to the approximate result shown in (26). The amplitude of the first contribution (diagram (a) in Fig. 2) can be written as

$$
\begin{gathered}
i \Gamma_{a}\left(p, k_{1}, k_{2}\right)=\int \frac{d^{4} Q}{(2 \pi)^{4}}\left(-i h_{\alpha 1}^{*} P_{L}\right) \frac{i}{\Phi-m_{\ell_{\alpha}}}\left(i \frac{m_{\ell_{\alpha}}}{v}\right) \frac{i}{Q+\not p-m_{\ell_{\alpha}}}\left(i h_{\alpha 1} P_{R}\right) \frac{i}{\left(k_{1}-Q\right)^{2}-m_{H^{+}}^{2}} \\
=h_{\alpha 1}^{*} h_{\alpha 1} \frac{m_{\ell_{\alpha}}}{v} \int \frac{d^{4} Q}{(2 \pi)^{4}} \frac{P_{L}\left(Q+m_{\ell_{\alpha}}\right)\left(Q+\not Q+m_{\ell_{\alpha}}\right) P_{R}}{\left[Q^{2}-m_{\ell_{\alpha}}^{2}\right]\left[(Q-p)^{2}-m_{\ell_{\alpha}}^{2}\right]\left[\left(k_{1}-Q\right)^{2}-m_{H^{+}}^{2}\right]} \\
=\frac{h_{\alpha 1}^{*} h_{\alpha 1} m_{\ell_{\alpha}}^{2}}{v} P_{L} \int \frac{d^{4} Q}{(2 \pi)^{4}} \frac{2 \not+\not p}{\left[Q^{2}-m_{\ell_{\alpha}}^{2}\right]\left[(Q-p)^{2}-m_{\ell_{\alpha}}^{2}\right]\left[\left(k_{1}-Q\right)^{2}-m_{H^{+}}^{2}\right]} .
\end{gathered}
$$

Using the Passarino-Veltman reduction [52], we find for the first contribution

$$
\begin{aligned}
\Gamma_{a}\left(p, k_{1}, k_{2}\right)= & \frac{1}{16 \pi^{2} v} \sum_{\alpha}\left|h_{\alpha 1}\right|^{2} m_{\ell_{\alpha}}^{2}\left\{C_{0}\left(M_{1}^{2}, p^{2}, M_{1}^{2}, m_{H^{ \pm}}^{2}, m_{\ell_{\alpha}}^{2}, m_{\ell_{\alpha}}^{2}\right)\right. \\
& \left.+C_{1}\left(M_{1}^{2}, p^{2}, M_{1}^{2}, m_{H^{ \pm}}^{2}, m_{\ell_{\alpha}}^{2}, m_{\ell_{\alpha}}^{2}\right)+C_{2}\left(M_{1}^{2}, p^{2}, M_{1}^{2}, m_{H^{ \pm}}^{2}, m_{\ell_{\alpha}}^{2}, m_{\ell_{\alpha}}^{2}\right)\right\} .
\end{aligned}
$$

The amplitude for the second contribution (diagram (b) in Fig. 2) is given by

$$
i \Gamma_{b}\left(p, k_{1}, k_{2}\right)=\int \frac{d^{4} Q}{(2 \pi)^{4}}\left(-i h_{\alpha 1}^{*} P_{L}\right) \frac{i}{\not k_{1}-\mathscr{Q}-m_{\ell_{\alpha}}}\left(i h_{\alpha 1} P_{R}\right) \frac{i}{(p+Q)^{2}-m_{H^{+}}^{2}}\left(i \lambda_{3} v\right) \frac{i}{Q^{2}-m_{H^{+}}^{2}}+\left(P_{L} \leftrightarrow P_{R}\right) .
$$

After some algebra, we get

$$
\Gamma_{b}\left(p, k_{1}, k_{2}\right)=-\frac{1}{16 \pi^{2}} \lambda_{3} v M_{1} \sum_{\alpha}\left|h_{\alpha 1}\right|^{2}\left\{C_{0}\left(p^{2}, M_{1}^{2}, M_{1}^{2}, m_{H^{ \pm}}^{2}, m_{H^{ \pm}}^{2}, m_{\ell_{\alpha}}^{2}\right)+C_{2}\left(p^{2}, M_{1}^{2}, M_{1}^{2}, m_{H^{ \pm}}^{2}, m_{H^{ \pm}}^{2}, m_{\ell_{\alpha}}^{2}\right)\right\} .
$$

The contribution of diagram (c) can be evaluated similarly to give

$$
\Gamma_{c}\left(p, k_{1}, k_{2}\right)=-\frac{1}{16 \pi^{2}}\left(\lambda_{3}+\lambda_{4} / 2\right) M_{1} v \sum_{S=H^{0}, A^{0}} \sum_{\alpha}\left|h_{\alpha 1}\right|^{2}\left\{C_{1}\left(p^{2}, M_{1}^{2}, M_{1}^{2}, 0, m_{S}^{2}, m_{S}^{2}\right)+C_{2}\left(p^{2}, M_{1}^{2}, M_{1}^{2}, 0, m_{S}^{2}, m_{S}^{2}\right)\right\},
$$

Numerically, we found that the contribution of diagram (a) is much smaller than the contribution of diagrams (b) and (c). Furthermore, in our numerical analysis, we use the expression quoted in (26) since it agrees very well with the full expression in terms of the Passarino-Veltman functions. 
[1] G. Aad et al. (ATLAS Collaboration), Observation of a new particle in the search for the Standard Model Higgs boson with the ATLAS detector at the LHC, Phys. Lett. B 716, 1 (2012); S. Chatrchyan et al. (CMS Collaboration), Observation of a new boson at a mass of $125 \mathrm{GeV}$ with the CMS experiment at the LHC, Phys. Lett. B 716, 30 (2012).

[2] A. Zee, Charged scalar field and quantum number violations, Phys. Lett. 161B, 141 (1985); E. Ma, Pathways to Naturally Small Neutrino Masses, Phys. Rev. Lett. 81, 1171 (1998).

[3] A. Zee, Quantum numbers of Majorana neutrino masses, Nucl. Phys. B264, 99 (1986); K. S. Babu, Model of "calculable" Majorana neutrino masses, Phys. Lett. B 203, 132 (1988); M. Aoki, S. Kanemura, T. Shindou, and K. Yagyu, An R-parity conserving radiative neutrino mass model without right-handed neutrinos, J. High Energy Phys. 07 (2010) 084; Erratum, 11 (2010) 049; G. Guo, X. G. He, and G. N. Li, Radiative two loop inverse seesaw and dark matter, J. High Energy Phys. 10 (2012) 044; Y. Kajiyama, H. Okada, and K. Yagyu, Two loop radiative seesaw model with inert triplet scalar field, Nucl. Phys. B874, 198 (2013).

[4] M. Aoki, S. Kanemura, and O. Seto, Neutrino Mass, Dark Matter and Baryon Asymmetry via TeV-Scale Physics without Fine-Tuning, Phys. Rev. Lett. 102, 051805 (2009); M. Aoki, S. Kanemura, and O. Seto, A model of $\mathrm{TeV}$ scale physics for neutrino mass, dark matter and baryon asymmetry and its phenomenology, Phys. Rev. D 80, 033007 (2009).

[5] L. M. Krauss, S. Nasri, and M. Trodden, A model for neutrino masses and dark matter, Phys. Rev. D 67, 085002 (2003).

[6] T. Nomura and H. Okada, Four-loop neutrino model inspired by diphoton excess at $750 \mathrm{GeV}$, Phys. Lett. B 755, 306 (2016).

[7] A. Ahriche, C. S. Chen, K. L. McDonald, and S. Nasri, Three-loop model of neutrino mass with dark matter, Phys. Rev. D 90, 015024 (2014).

[8] A. Ahriche, K. L. McDonald, and S. Nasri, A model of radiative neutrino mass: With or without dark matter, J. High Energy Phys. 10 (2014) 167.

[9] A. Ahriche, K. L. McDonald, S. Nasri, and T. Toma, A model of neutrino mass and dark matter with an accidental symmetry, Phys. Lett. B 746, 430 (2015).

[10] A. Ahriche, K. L. McDonald, and S. Nasri, A radiative model for the weak scale and neutrino mass via dark matter, J. High Energy Phys. 02 (2016) 038.

[11] A. Ahriche, S. Nasri, and R. Soualah, Radiative neutrino mass model at the $e^{-} e^{+}$linear collider, Phys. Rev. D 89, 095010 (2014); C. Guella, D. Cherigui, A. Ahriche, S. Nasri, and R. Soualah, Probing radiative neutrino mass models with dilepton events at the LHC, Phys. Rev. D 93, 095022 (2016); D. Cherigui, C. Guella, A. Ahriche, and S. Nasri, Probing radiative neutrino mass models using trilepton channel at the LHC, Phys. Lett. B 762, 225 (2016); M. Chekkal, A. Ahriche, A. B. Hammou, and S. Nasri, Right-handed neutrinos: dark matter, lepton flavor violation and leptonic collider searches, Phys. Rev. D 95, 095025 (2017).
[12] K. Cheung and O. Seto, Phenomenology of charged scalars in the $C P$-violating inert-doublet model, Phys. Rev. D 69, 113009 (2004).

[13] Y. Cai, J. Herrero-García, M. A. Schmidt, A. Vicente, and R. R. Volkas, From the trees to the forest: a review of radiative neutrino mass models, Front. Phys. 5, 63 (2017).

[14] A. Ahriche, and S. Nasri, Dark matter and strong electroweak phase transition in a radiative neutrino mass model, J. Cosmol. Astropart. Phys. 07 (2013) 035, A. Ahriche, K. L. McDonald, and S. Nasri, Scalar sector phenomenology of three-loop radiative neutrino mass models, Phys. Rev. D 92, 095020 (2015).

[15] N. G. Deshpande and E. Ma, Pattern of symmetry breaking with two Higgs doublets, Phys. Rev. D 18, 2574 (1978); R. Barbieri, L. J. Hall, and V. S. Rychkov, Improved naturalness with a heavy Higgs: An Alternative road to LHC physics, Phys. Rev. D 74, 015007 (2006).

[16] M. Gustafsson, E. Lundstrom, L. Bergstrom, and J. Edsjo, Significant Gamma Lines from Inert Higgs Dark Matter, Phys. Rev. Lett. 99, 041301 (2007); T. Hambye and M. H. G. Tytgat, Electroweak symmetry breaking induced by dark matter, Phys. Lett. B 659, 651 (2008); P. Agrawal, E. M. Dolle, and C. A. Krenke, Signals of inert doublet dark matter in neutrino telescopes, Phys. Rev. D 79, 015015 (2009); E. M. Dolle and S. Su, The inert dark matter, Phys. Rev. D 80, 055012 (2009); E. Dolle, X. Miao, S. Su, and B. Thomas, Dilepton signals in the inert doublet model, Phys. Rev. D 81, 035003 (2010); S. Andreas, M. H. G. Tytgat, and Q. Swillens, Neutrinos from inert doublet dark matter, J. Cosmol. Astropart. Phys. 04 (2009) 004; D. Borah and J. M. Cline, Inert doublet dark matter with strong electroweak phase transition, Phys. Rev. D 86, 055001 (2012); E. Nezri, M. H. G. Tytgat, and G. Vertongen, e+ and anti-p from inert doublet model dark matter, J. Cosmol. Astropart. Phys. 04 (2009) 014; I. F. Ginzburg, K. A. Kanishev, M. Krawczyk, and D. Sokolowska, Evolution of Universe to the present inert phase, Phys. Rev. D 82, 123533 (2010); X. Miao, S. $\mathrm{Su}$, and B. Thomas, Trilepton signals in the inert doublet model, Phys. Rev. D 82, 035009 (2010); M. Gustafsson, S. Rydbeck, L. Lopez-Honorez, and E. Lundstrom, Status of the inert doublet model and the role of multileptons at the LHC, Phys. Rev. D 86, 075019 (2012); A. Arhrib, R. Benbrik, and N. Gaur, $H \rightarrow \gamma \gamma$ in inert Higgs doublet model, Phys. Rev. D 85, 095021 (2012); A. Arhrib, Y. L. S. Tsai, Q. Yuan, and T. C. Yuan, An updated analysis of inert Higgs doublet model in light of the recent results from LUX, PLANCK, AMS-02 and LHC, J. Cosmol. Astropart. Phys. 06 (2014) 030; A. Goudelis, B. Herrmann, and O. Stål, Dark matter in the inert doublet model after the discovery of a Higgs-like boson at the LHC, J. High Energy Phys. 09 (2013) 106; A. Arhrib, R. Benbrik, and T. C. Yuan, Associated production of Higgs at linear collider in the inert Higgs doublet model, Eur. Phys. J. C 74, 2892 (2014); G. Belanger, B. Dumont, A. Goudelis, B. Herrmann, S. Kraml, and D. Sengupta, Dilepton constraints in the inert doublet model from Run 1 of the LHC, Phys. Rev. D 91, 115011 (2015); A. Arhrib, R. Benbrik, J. El Falaki, and A. Jueid, Radiative corrections to the triple Higgs coupling in the inert Higgs doublet model, J. High Energy Phys. 12 (2015) 007; S. Banerjee and N. Chakrabarty, A revisit to 
scalar dark matter with radiative corrections, arXiv: 1612.01973; S. Kanemura, M. Kikuchi, and K. Sakurai, Testing the dark matter scenario in the inert doublet model by future precision measurements of the Higgs boson couplings, Phys. Rev. D 94, 115011 (2016); P. Poulose, S. Sahoo, and K. Sridhar, Exploring the Inert Doublet Model through the dijet plus missing transverse energy channel at the LHC, Phys. Lett. B 765, 300 (2017); F. P. Huang and J. H. Yu, Explore inert dark matter blind spots with gravitational wave signatures, arXiv:1704.04201; A. Vicente and C. E. Yaguna, Probing the scotogenic model with lepton flavor violating processes, J. High Energy Phys. 02 (2015) 144; F. S. Queiroz and C. E. Yaguna, The CTA aims at the inert doublet model, J. Cosmol. Astropart. Phys. 02 (2016) 038; A. Alves, D. A. Camargo, A. G. Dias, R. Longas, C. C. Nishi, and F. S. Queiroz, Collider and dark matter searches in the inert doublet model from PecceiQuinn symmetry, J. High Energy Phys. 10 (2016) 015; T. A. Chowdhury, M. Nemevsek, G. Senjanovic, and Y. Zhang, Dark matter as the trigger of strong electroweak phase transition, J. Cosmol. Astropart. Phys. 02 (2012) 029.

[17] S. L. Glashow and S. Weinberg, Natural conservation laws for neutral currents, Phys. Rev. D 15, 1958 (1977).

[18] A. Ahriche, K. L. McDonald, and S. Nasri, The scaleinvariant scotogenic model, J. High Energy Phys. 06 (2016) 182.

[19] D. Borah, S. Sadhukhan, and S. Sahoo, Lepton portal limit of inert Higgs doublet dark matter with radiative neutrino mass, Phys. Lett. B 771, 624 (2017).

[20] L. L. Honorez and C. E. Yaguna, The inert doublet model of dark matter revisited, J. High Energy Phys. 09 (2010) 046.

[21] S. Choubey and A. Kumar, Inflation and dark matter in the inert doublet model, J. High Energy Phys. 11 (2017) 080.

[22] E. Ma, Verifiable radiative seesaw mechanism of neutrino mass and dark matter, Phys. Rev. D 73, 077301 (2006).

[23] S. P. Martin, Two loop effective potential for a general renormalizable theory and softly broken supersymmetry, Phys. Rev. D 65, 116003 (2002).

[24] A. Merle and M. Platscher, Parity problem of the scotogenic neutrino model, Phys. Rev. D 92, 095002 (2015).

[25] B. Pontecorvo, Neutrino experiments and the problem of conservation of leptonic charge, Zh. Eksp. Teor. Fiz. 53, 1717 (1967) [Sov. Phys. JETP 26, 984 (1968)].

[26] D. V. Forero, M. Tortola, and J. W. F. Valle, Global status of neutrino oscillation parameters after Neutrino-2012, Phys. Rev. D 86, 073012 (2012).

[27] K. G. Klimenko, Teor. Mat. Fiz. 62, 87 (1985) [Conditions for certain Higgs potentials to be bounded below, Theor. Math. Phys. 62, 58 (1985)].

[28] I. F. Ginzburg, K. A. Kanishev, M. Krawczyk, and D. Sokolowska, Evolution of Universe to the present inert phase, Phys. Rev. D 82, 123533 (2010); B. Swiezewska, Yukawa independent constraints for two-Higgs-doublet models with a $125 \mathrm{GeV}$ Higgs boson, Phys. Rev. D 88, 055027 (2013); Erratum, 88, 119903 (2013).

[29] A. G. Akeroyd, A. Arhrib, and E. M. Naimi, Note on tree level unitarity in the general two Higgs doublet model, Phys. Lett. B 490, 119 (2000).

[30] T. Toma and A. Vicente, Lepton flavor violation in the scotogenic model, J. High Energy Phys. 01 (2014) 160.
[31] C. Patrignani et al. (Particle Data Group), Review of particle physics, Chin. Phys. C 40, 100001 (2016). A. M. Baldini et al. (MEG Collaboration), Search for the lepton flavour violating decay $\mu^{+} \rightarrow \mathrm{e}^{+} \gamma$ with the full dataset of the MEG experiment, Eur. Phys. J. C 76, 434 (2016); B. Aubert et al. (BABAR Collaboration), Searches for Lepton Flavor Violation in the Decays $\tau \pm \rightarrow e \pm \gamma$ and $\tau \pm \rightarrow \mu \pm \gamma$, Phys. Rev. Lett. 104, 021802 (2010); K. Hayasaka et al., Search for lepton flavor violating tau decays into three leptons with 719 million produced $\tau+\tau$ - pairs, Phys. Lett. B 687, 139 (2010); U. Bellgardt et al. (SINDRUM Collaboration), Search for the decay $\mu+\rightarrow e+e+e-$, Nucl. Phys. B299, 1 (1988).

[32] W. Grimus, L. Lavoura, O. M. Ogreid, and P. Osland, The oblique parameters in multi-Higgs-doublet models, Nucl. Phys. B801, 81 (2008).

[33] The ATLAS collaboration, Report No. ATLAS-CONF2017-047.

[34] C. S. Chen, C. Q. Geng, D. Huang, and L. H. Tsai, New scalar contributions to $h \rightarrow Z \gamma$, Phys. Rev. D 87, 075019 (2013).

[35] J. Abdallah et al. (DELPHI Collaboration), Searches for supersymmetric particles in $e^{+} e^{-}$collisions up to $208-\mathrm{GeV}$ and interpretation of the results within the MSSM, Eur. Phys. J. C 31, 421 (2003); M. Acciarri et al. (L3 Collaboration), Search for charginos and neutralinos in $e^{+} e^{-}$ collisions at $\sqrt{S}=189-\mathrm{GeV}$, Phys. Lett. B 472, 420 (2000); R. Barate et al. (ALEPH Collaboration), Search for supersymmetric particles in $e^{+} e^{-}$collisions at $\sqrt{s}$ up to 202-GeV and mass limit for the lightest neutralino, Phys. Lett. B 499, 67 (2001); G. Abbiendi et al. (OPAL Collaboration), Search for chargino and neutralino production at $s * *(1 / 2)=192-\mathrm{GeV}$ to $209 \mathrm{GeV}$ at LEP, Eur. Phys. J. C 35, 1 (2004).

[36] E. Lundstrom, M. Gustafsson, and J. Edsjo, The inert doublet model and LEP II limits, Phys. Rev. D 79, 035013 (2009).

[37] P. Gondolo and G. Gelmini, Cosmic abundances of stable particles: Improved analysis, Nucl. Phys. B360, 145 (1991).

[38] G. Jungman, M. Kamionkowski, and K. Kong, Supersymmetric dark matter, Phys. Rep. 267, 195 (1996).

[39] G. Hinshaw et al. (WMAP Collaboration), Nine-Year Wilkinson Microwave Anisotropy Probe (WMAP) observations: Cosmological parameter results, Astrophys. J. Suppl. Ser. 208, 19 (2013).

[40] P. A. R. Ade et al. (Planck Collaboration), Planck 2015 results. XIII. Cosmological parameters, Astron. Astrophys. 594, A13 (2016).

[41] K. Kong and K. T. Matchev, Precise calculation of the relic density of Kaluza-Klein dark matter in universal extra dimensions, J. High Energy Phys. 01 (2006) 038.

[42] X. G. He, T. Li, X. Q. Li, J. Tandean, and H. C. Tsai, Constraints on scalar dark matter from direct experimental searches, Phys. Rev. D 79, 023521 (2009).

[43] N. Okada and T. Yamada, Simple fermionic dark matter models and Higgs boson couplings, J. High Energy Phys. 10 (2013) 017.

[44] D. S. Akerib et al. (LUX Collaboration), Results from a Search for Dark Matter in the Complete LUX Exposure, Phys. Rev. Lett. 118, 021303 (2017). 
[45] E. Aprile et al. (XENON Collaboration), First Dark Matter Search Results from the XENON1T Experiment, Phys. Rev. Lett. 119, 181301 (2017).

[46] A. Belyaev, N. D. Christensen, and A. Pukhov, CalcHEP 3.4 for collider physics within and beyond the standard model, Comput. Phys. Commun. 184, 1729 (2013).

[47] J. Alwall, M. Herquet, F. Maltoni, O. Mattelaer, and T. Stelzer, MadGraph 5: Going beyond, J. High Energy Phys. 06 (2011) 128.

[48] T. Sjostrand, S. Mrenna, and P.Z. Skands, A brief introduction to PYTHIA 8.1, Comput. Phys. Commun. 178, 852 (2008).

[49] A. Ahriche, A. Arhrib, A. Jueid, S. Nasri, and A. de la Puente (to be published).

[50] T. Hahn, Generating Feynman diagrams and amplitudes with FeynArts 3, Comput. Phys. Commun. 140, 418 (2001); T. Hahn and C. Schappacher, The implementation of the
Minimal Supersymmetric Standard Model in FeynArts and FormCalc, Comput. Phys. Commun. 143, 54 (2002); T. Hahn and M. Perez-Victoria, Automated one-loop calculations in four and $D$ dimensions, Comput. Phys. Commun. 118, 153 (1999); J. Küblbeck, M. Böhm, and A. Denner, Feyn arts - computer-algebraic generation of Feynman graphs and amplitudes, Comput. Phys. Commun. 60, 165 (1990).

[51] G. J. van Oldenborgh, FF-a package to evaluate one-loop Feynman diagrams, Comput. Phys. Commun. 66, 1 (1991); T. Hahn, Acta Phys. Polon. B 30, 3469 (1999), Feynman diagram calculations with FeynArts, FormCalc, and LoopTools, Proc. Sci., ACAT2010 (2010) 078, [arXiv:1006.2231].

[52] G. Passarino and M. J. G. Veltman, One loop corrections for $e^{+} e^{-}$annihilation into $\mu^{+} \mu^{-}$in the Weinberg model, Nucl. Phys. B160, 151 (1979). 